From the ${ }^{1}$ Department of Biochemistry and Molecular Biology; ${ }^{2}$ Program in Molecular and Cellular Biology; University of Massachusetts, Amherst, MA, 01003

${ }^{3}$ Department of Cellular and Molecular Medicine, University of Copenhagen, Copenhagen, Denmark. ${ }^{4}$ Copenhagen Center for Glycomics, University of Copenhagen, Copenhagen, Denmark.

\title{
Quantitative glycoproteomics reveals cellular substrate selectivity of the ER protein quality control sensors UGGT1 and UGGT2
}

\author{
*To whom correspondence should be addressed: Daniel N. Hebert, Department of Biochemistry \\ and Molecular Biology, University of Massachusetts, 240 Thatcher Road Amherst, MA 01003, Tel: \\ (413) 545-0079 Fax: (413) 545-3291, E-mail: dhebert@biochem.umass.edu
}

\section{Keywords}

calnexin, calreticulin, lectin chaperones, protein homeostasis, glycoproteomics

\section{ABSTRACT}

UDP-glucose: glycoprotein glucosyltransferase (UGGT) 1 and 2 are central hubs in the chaperone network of the endoplasmic reticulum (ER), acting as gatekeepers to the early secretory pathway yet little is known about their cellular clients. These two quality control sensors control lectin chaperone binding and glycoprotein egress from ER. A quantitative glycoproteomics strategy was deployed to identify cellular substrates of the UGGTs at endogenous levels in CRISPR-edited HEK293 cells. The seventy-one UGGT substrates identified were mainly large multidomain and heavily glycosylated proteins when compared to the general N-glycome. UGGT1 was the dominant glucosyltransferase with a preference towards large plasma membrane proteins whereas UGGT2 favored the modification of smaller, soluble lysosomal proteins. This study sheds light on differential specificities and roles of UGGT1 and UGGT2 and provides insight into the cellular reliance on carbohydrate-dependent chaperone

31 intervention by UGGT1 and UGGT2 to facilitate proper folding and maturation of the cellular N-

32 glycome. 


\section{INTRODUCTION}

2 Protein folding in the cell is an error-prone process and protein misfolding is the basis for a

3 large number of disease states (Hebert and Molinari, 2007; Hartl, 2017). A significant fraction of

4 the proteome in mammalian cells passes through the secretory pathway by first being targeted

5 to the endoplasmic reticulum (ER) where folding occurs (Uhlén et al., 2015; Itzhak et al., 2016;

6 Adams et al., 2019a). Molecular chaperones of the ER help to guide secretory pathway cargo

7 along a productive folding pathway by directing the trajectory of the folding reaction, inhibiting

8 non-productive side reactions such as aggregation or by retaining immature or misfolded

9 proteins in the ER until they can properly fold or be targeted for degradation. Understanding

10 how chaperone binding controls the maturation and flux of proteins through the secretory

11 pathway is of important fundamental biological concern and will impact our knowledge of

12 protein folding diseases and the development of potential therapeutics including the

13 production of biologics that are frequently secretory proteins.

Proteins that traverse the secretory pathway are commonly modified with $\mathrm{N}$-linked

15 glycans as they enter the ER lumen (Zielinska et al., 2010). These carbohydrates serve a variety

16 of roles including acting as quality control tags or attachment sites for the lectin ER chaperones

17 calnexin and calreticulin (Helenius and Aebi, 2004; Hebert et al., 2014). N-glycosylation

18 commences co-translationally in mammals and the first round of binding to calnexin and

19 calreticulin is initiated shortly thereafter by the rapid trimming of glucoses by glucosidases I and

20 II to reach their monoglucosylated state (Chen et al., 1995; Cherepanova et al., 2019). Lectin

21 chaperone binding is multifunctional as it has been shown to: (1) direct the folding trajectory of

22 a protein by acting as a holdase that slows folding in a region-specific manner; (2) act as an

23 adapter or platform to recruit folding factors including oxidoreductases (ERp57 and ERp29) and

24 a peptidyl-prolyl cis trans isomerase (CypB) to maturing nascent chains; (3) diminish

25 aggregation; (4) retain immature, misfolded or unassembled proteins in the ER; and (5) target

26 aberrant proteins for degradation (Rajagopalan et al., 1994; Hebert et al., 1996; Daniels et al.,

27 2003; Molinari et al., 2003; Oda et al., 2003; Wang et al., 2008; Kozlov and Gehring, 2020). For

28 glycoproteins, the lectin chaperones appear to be the dominant chaperone system as once an

$29 \mathrm{~N}$-glycan is added to a region on a protein, it has been shown to be rapidly passed from the ER 
1 Hsp70 chaperone BiP to the lectin chaperones, further underscoring their central role in controlling protein homeostasis in the secretory pathway (Helenius and Hammond, 1994).

$\mathrm{N}$-glycan trimming to an unglucosylated glycoform by glucosidase II supports substrate release from the lectin chaperones. At this stage, if the protein folds properly, it is packaged into COPII vesicles for anterograde trafficking (Barlowe and Helenius, 2016). Alternatively, substrates that are evaluated to be non-native are directed for rebinding to the lectin chaperones by the protein folding sensor UDP-glucose: glycoprotein glucosyltransferase 1 (UGGT1) that reglucosylates immature or misfolded proteins (Helenius, 1994; Sousa and Parodi, 1995). Since UGGT1 directs the actions of this versatile lectin chaperone system and thereby controls protein trafficking through the ER, it acts as a key gatekeeper of the early secretory pathway. Therefore, it is vital to understand the activity of UGGT1 and the scope of substrates

12 it modifies.

Our current knowledge of the activity of UGGT1 relies largely on studies using purified components. UGGT1 was found to recognize non-native or near-native glycoproteins with exposed hydrophobic regions using in vitro approaches where the modification of

16 glycopeptides, engineered or model substrates by purified UGGT1 was monitored (Ritter and 17 Helenius, 2000; Taylor et al., 2003; Caramelo et al., 2004). Recent crystal structures of fungal

18 UGGT1 have shown that it possesses a central, hydrophobic cavity in its protein sensing domain, which may support hydrophobic-based interactions for substrate selection (Roversi et al., 2017; Satoh et al., 2017).

Cell-based studies of UGGT1 have relied on the overexpression of cellular and viral proteins (Soldà et al., 2007; Pearse et al., 2008; Ferris et al., 2013; Tannous et al., 2015). Uggt1 knockout studies have found that the roles of UGGT1 appear to be substrate specific as UGGT1 can promote, decrease or not affect the interaction between substrates and calnexin (Soldà et al., 2007). Prosaposin, the only known cellular substrate of UGGT1 when expressed at endogenous levels, grossly misfolds in the absence of Uggt1 and accumulates in aggresome-like structures (Pearse et al., 2010). Work in animals has further emphasized the importance of UGGT1 as the deletion of Uggt1 in mice is embryonically lethal (Molinari et al., 2005). 
UGGT1 has a paralogue, UGGT2, but it has no demonstrated cellular activity (Arnold et al., 2000). Domain swapping experiments have demonstrated that UGGT2 possesses a catalytically active glucosyltransferase domain when appended to the folding sensor domain of UGGT1 (Arnold and Kaufman, 2003). In vitro experiments using purified, chemically glycosylated interleukin-8 (IL-8), which is not glycosylated in cells, have found that UGGT2 can glucosylate IL-8 (Takeda et al., 2014). This suggests that UGGT2 may be an additional reglucosylation enzyme or protein folding sensor of the ER.

Unlike the classical ATP-dependent chaperones that directly query the conformation of their substrates (Balchin et al., 2016), binding to the lectin chaperones is dictated by enzymes that covalently modify the substrate (Helenius and Aebi, 2004; Hebert et al., 2014). Rebinding

11 to the carbohydrate-dependent chaperones is initiated by the UGGTs that interrogate the 12 integrity of the structure of the protein. Therefore, the proteome-wide detection of cellular

13 UGGT substrates provides the unprecedented opportunity to identify clients that require 14 multiple rounds of chaperone binding and are more reliant on lectin chaperone binding for 15 proper maturation and sorting. Therefore, we designed a cell-based quantitative 16 glycoproteomics approach to identify high-confidence endogenous substrates of UGGT1 and UGGT2 by the affinity purification of monoglucosylated substrates in CRISPR/Cas9-edited cells.

18 UGGT1 and UGGT2 substrates were found to display multiple features of complex proteins including extended lengths plus large numbers of Cys residues and N-glycans. Specific substrates of either UGGT1 or UGGT2 were also discovered, therefore determining that UGGT2 possessed glucosyltransferase activity and identifying its first natural substrates. UGGT1 demonstrated a slight preference for transmembrane proteins, especially those targeted to the plasma membrane, while UGGT2 modification favored soluble lysosomal proteins. The identification of reglucosylated substrates improves our understanding of their folding and maturation pathways and has implications regarding how folding trajectories may be altered in 26 disease states.

\section{RESULTS}


1 To identify the substrates that are most dependent upon persistent calnexin/calreticulin cycle

2 binding, we isolated and identified endogenous substrates for the ER protein folding sensors

3 UGGT1 and UGGT2. As the product of a reglucosylation by the UGGTs is a monoglucosylated N-

4 glycan, the presence of the monoglucosylated glycoform was used as a readout for substrate

5 reglucosylation. $\mathrm{N}$-glycans are originally transferred to nascent glycoproteins containing three

6 glucoses, therefore a monoglucosylated glycan can be generated either through trimming of

7 two glucoses from the nascent $\mathrm{N}$-linked glycan or through reglucosylation by the UGGTs. In

8 order to isolate the reglucosylation step from the trimming process, a gene edited cell line was

9 created that transfers abbreviated unglucosylated N-linked glycans to nascent chains. The N-

10 linked glycosylation pathway in mammalian cells is initiated through the sequential addition of

11 monosaccharides, mediated by the $A L G$ (Asn-linked glycosylation) gene products, to a

12 cytosolically exposed dolichol-P-phosphate embedded in the ER membrane (Aebi, 2013;

13 Cherepanova et al., 2016) (Figure 1A). The immature dolichol-P-phosphate precursor is then

14 flipped into the ER lumen and sequential carbohydrate addition is continued by additional ALG

15 proteins. The completed N-glycan $\left(\mathrm{Glc}_{3} \mathrm{Man}_{9} \mathrm{GlcNAc}_{2}\right)$ is then appended to an acceptor Asn

16 residue in the sequon Asn-Xxx-Ser/Thr/Cys (where Xxx is not a Pro) by the oligosaccharyl

17 transferase (OST) complex (Cherepanova et al., 2016). Initially, a Chinese Hamster Ovary (CHO)

18 cell line with a defect in Alg6 was employed to establish the utility of this approach to follow

19 (re)glucosylation (Quellhorst et al., 1999; Cacan et al., 2001; Pearse et al., 2008, 2010; Tannous

20 et al., 2015). As the CHO proteome is poorly curated compared to the human proteome,

21 CRISPR/Cas9 was used to knock-out the ALG6 gene in HEK293EBNA1-6E cells to provide a

22 cellular system that transferred non-glucosylated glycans (Man, $\mathrm{GlcNAc}_{2}$ ) to substrates. In these

$23 A L G 6^{-/}$cells, a monoglucosylated glycan is solely created by the glucosylation by the UGGTs

24 providing a suitable system to follow the glucosylation process (Figure 1B).

To aid in substrate identification, an inhibitor of glucosidases I and II, deoxynojirimycin

26 (DNJ), was added $1 \mathrm{hr}$ prior to cell lysis to block glucose trimming and trap monoglucosylated

27 products. Monoglucosylated substrates were then isolated by affinity purification using

28 recombinant glutathione S-transferase-calreticulin (GST-CRT), as calreticulin binds

29 monoglucosylated proteins. To account for non-specific binding, a lectin-deficient construct 
1 (GST-CRT-Y109A) was used as an affinity purification control (Kapoor et al., 2004). Affinity

2 purified substrates were reduced, alkylated, and trypsin digested. The resulting peptides were

3 labeled with tandem mass tags (TMT) (Rauniyar and Yates, 2014), deglycosylated using

4 PNGaseF, and analyzed by mass spectrometry to identify substrates of the UGGTs. The use of

5 TMT, as well as the control GST-CRT-Y109A affinity purification, allows for robust, quantitative

6 identification of substrates of the UGGTs. The resulting data was analyzed by calculating the

7 fold change in abundance of the TMT associated with proteins identified through affinity

8 purification using wild type GST-CRT over affinity purification using GST-CRT-Y109A. To be

9 considered a UGGT substrate, a cutoff of three-fold (wild type GST-CRT/GST-CRT-Y109A) was

10 applied. This conservative cutoff was set to give a high level of confidence in the identified

11 substrates, as below this cutoff, increasing fractions of non-secretory pathway proteins were

12 found.

13

14

15

16

17

18 
Figure 1

A

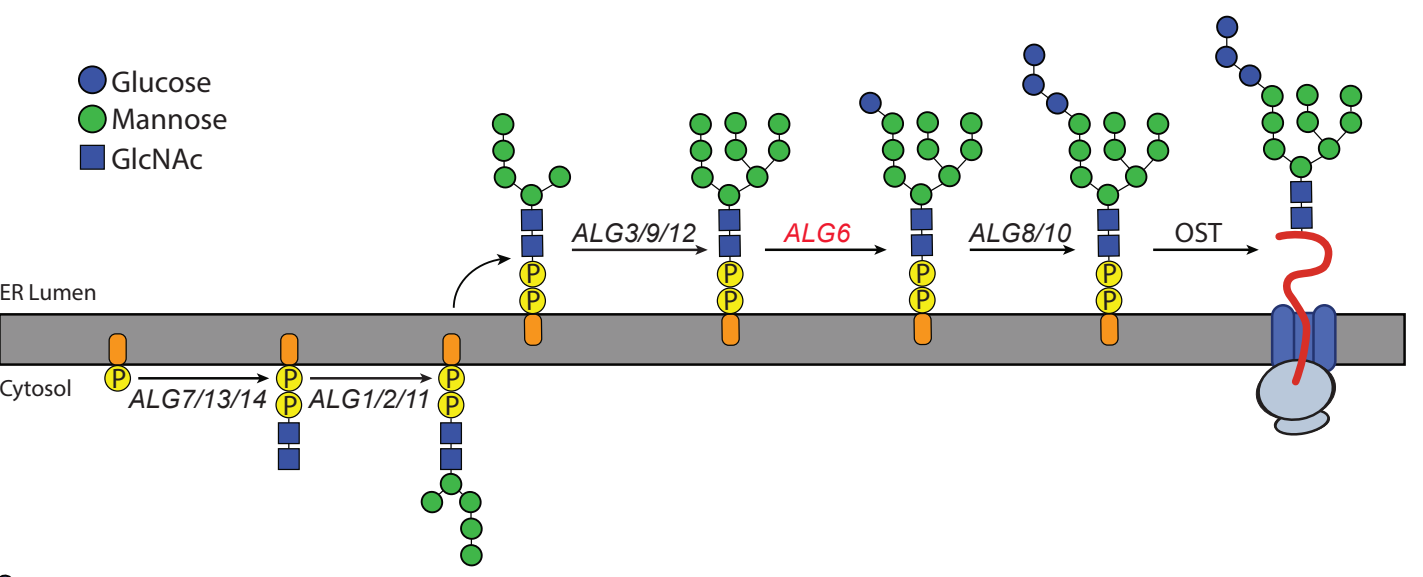

B

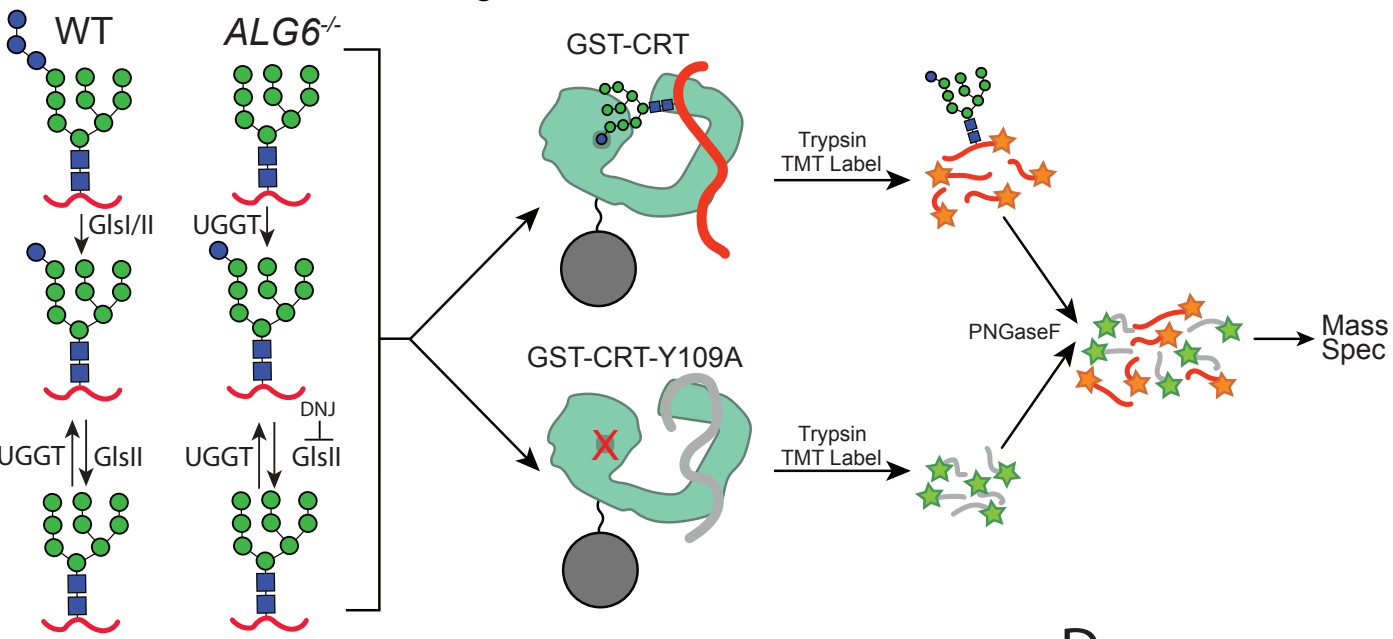

C
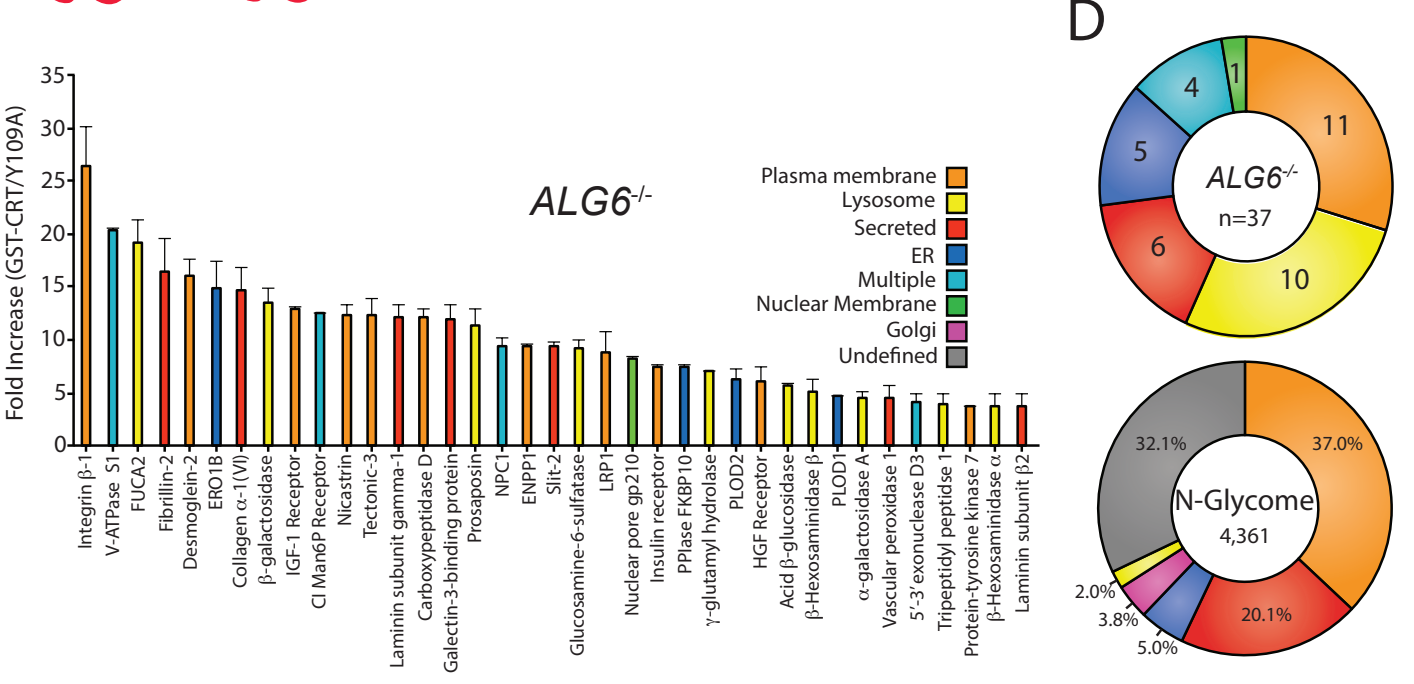

2 Figure 1. The identification of UGGT1/2 substrates.

3 (A) The pathway of $\mathrm{N}$-glycosylation in eukaryotic cells is depicted. $\mathrm{N}$-glycan synthesis is initiated

4 in the outer ER membrane leaflet on a dolichol-P-phosphate facing the cytoplasm. Flipping of 
1 the precursor $\mathrm{N}$-glycan to the ER luminal leaflet and further synthesis steps mediated by ALG

2 proteins leads to eventual transfer of a $\mathrm{Glc}_{3} \mathrm{Man}_{9} \mathrm{GlcNAc}_{2} \mathrm{~N}$-glycan to a substrate by the OST

3 complex. ALG6 (red lettering) catalyzes the transfer of the initial glucose onto the Mang

4 precursor N-glycan. (B) In wild type (WT) cells, a Glc $3 M_{3} \operatorname{Man}_{9} \mathrm{GlNAc}_{2} \mathrm{~N}$-glycan is transferred to

5 substrates. Monoglucosylated substrates may therefore occur via trimming by glucosidases I/II

6 (GIsI/II) or reglucosylation by UGGT1/2. In $\mathrm{ALG6}^{-/}$cells, a Man ${ }_{9} \mathrm{GICNAc}_{2} \mathrm{~N}$-glycan is transferred

7 to substrates. Therefore, monoglucosylated substrates may only occur through reglucosylation

8 by UGGT1/2. DNJ $(500 \mu \mathrm{M})$ was added to block the trimming of monoglucosylated substrates

9 by GlsIl. ALG6\% cells were then lysed and split equally between affinity purifications with either

10 GST-CRT or GST-CRT-Y109A bound to glutathione beads. Affinity-purified samples were then

11 reduced, alkylated, trypsinzed, and labeled with TMT labels. Samples were then deglycosylated

12 with PNGaseF, pooled, and analyzed by mass spectrometry. (C) Substrates were identified by

13 dividing the quantification of the TMT label in the GST-CRT condition for each protein by that of

14 the associated GST-CRT-Y109A condition, yielding the fold increase. Localization as predicted by

15 Uniprot annotation is depicted. A cutoff of three-fold increase was applied. Data is

16 representative of two independent experiments. Error bars represent standard error of the

17 mean (SEM). (D) The N-glycome was computationally determined by collecting all proteins

18 annotated to contain an N-glycome by Uniprot. Annotated localization information was then

19 used to computationally determine the localization distribution of the N-glycome as well as the

20 identified UGGT substrates. 


\section{Substrate identification of the UGGTs}

In order to determine the cellular substrates of the UGGTs, the above glycoproteomics protocol was followed using $A L G 6^{\%}$ cells. A restricted pool of thirty-seven $\mathrm{N}$-linked glycosylated proteins was identified as substrates of the UGGTs (Figure 1C, Supplemental Table 1). Prosaposin, the only previously known endogenous substrate of the UGGTs, was included in this group, supporting the utility of the approach (Pearse et al., 2010). Integrin $\beta-1$ showed the most significant fold change (wild type GST-CRT/GST-CRT-Y109A) of 26-fold, indicating there is a large dynamic range of reglucosylation levels.

The cell localizations of UGGT substrates were then determined by using their Uniprot classification. Approximately two thirds of the UGGT substrates are destined for the plasma

11 membrane or lysosomes (Figure 1C and D). Additional substrates are secreted or are resident to 12 the ER or nuclear membrane. Nuclear pore membrane glycoprotein 210 (NUP210) was the only 13 nuclear membrane protein found to be reglucosylated and it is the sole subunit of the nuclear 14 pore that is $\mathrm{N}$-glycosylated (Beck and Hurt, 2016). The nucleus and ER share a contiguous membrane. Proteins targeted to the nuclear membrane are first inserted into the ER

16 membrane, then move laterally to the nuclear membrane (Katta et al., 2014). Four proteins

17 were designated as 'multiple localizations' including cation-independent mannose-6-phosphate 18 receptor (Cl-M6PR), which traffics between the Golgi, lysosome and plasma membrane 19 (Dell'Angelica and Payne, 2001).

To distinguish the general pool of substrates that the UGGTs are expected to be exposed to, $\mathrm{N}$-glycosylated proteins of the secretory pathway proteome (N-glycome) were

22 computationally defined (Supplemental Table 2). The N-glycome is comprised of proteins that

23 are targeted to the ER either for residency in the secretory/endocytic pathways or for

24 trafficking to the plasma membrane or for secretion. The reviewed UniprotKB $H$. sapiens

25 proteome (20,353 total proteins) was queried to identify all proteins annotated as $\mathrm{N}$ -

26 glycosylated, resulting in a set of 4,520 proteins. This set was then curated to remove proteins

27 predicted to be mitochondrial, contain less than 50 amino acids or redundant isoforms. The

28 resulting $\mathrm{N}$-glycome contained 4,361 proteins, predicting $~ 21 \%$ of the proteome is $\mathrm{N}$ - 
1 glycosylated. Comparing UGGT substrates to the N-glycome allows for the characterization of feature preferences of substrates for the UGGTs.

The majority of the $\mathrm{N}$-glycome was either localized to the plasma membrane $(37 \%)$ or was secreted (20\%) according to their Uniprot designations. Smaller fractions of the N-glycome reside in the ER (5\%), Golgi (4\%) or lysosomes (2\%). UGGT substrates are therefore significantly enriched for lysosomal proteins compared to the N-glycome, while all other localizations display a similar distribution to their availability. In total, these results demonstrate the ability to identify substrates of the UGGTs proteomically and suggest that the UGGTs display substrate

9 preferences.

\section{Determination of UGGT1 and UGGT2 specific substrates}

12 There are two ER glucosyltransferase paralogues, UGGT1 and UGGT2, though currently there is 13 no evidence that UGGT2 acts as a protein sensor or a glucosyltransferase in the cell. Therefore, 14 we sought to determine if UGGT2 has glucosyltransferase activity in the cell, and if so, do these two paralogues have different substrate specificities. To address this concern, GST-CRT affinity

16 purification and TMT mass spectrometry were used to identify substrates of UGGT1 in

17 ALG6/UGGT2\% cells and potential UGGT2 substrates in ALG6/UGGT1\% cells.

With the ALG6/UGGT2\%- cells, $66 \mathrm{~N}$-glycosylated proteins were identified as reglucosylation substrates using the three-fold cutoff (GST-CRT/CST-CRT-Y109A) (Figure 2A).

20 Nearly double the number of UGGT1 substrates were identified through this approach compared to using $A L G 6^{-/}$cells where both UGGT1 and UGGT2 were present. This expansion in substrate number is likely due to the $~ 50 \%$ increase in expression of UGGT1 in ALG6/UGGT2\%cells (Supplemental Figure 1). The substrate demonstrating the most significant fold change (23.5-fold) was CD164, creating a similar dynamic range for reglucosylation to that observed in ALG6\% cells.

To identify possible UGGT2 specific substrates, ALG6/UGGT1\% cells were used to isolate

28 Y109A cutoff, with 33 of these proteins predicted to be N-glycosylated and localized to the secretory pathway (Figure 2B). Importantly, this demonstrated for the first time that UGGT2 
1 was a functional glycosyltransferase capable of reglucosylating a range of cellular substrates.

2 The glycoprotein with the most significant fold change was arylsulfatase A (10.4-fold). Notably,

38 of the 9 strongest UGGT2 substrates or, 15 of 33 substrates overall, are lysosomal proteins

4 (Figure 2B and C). While UGGT1 was also observed to engage a significant percentage of

5 lysosomal proteins (27\%), $45 \%$ of UGGT2 substrates are lysosomal. Both of these percentages

6 are significantly enriched when compared to the $\mathrm{N}$-glycome for which only $2 \%$ is comprised of

7 resident lysosome proteins (Figure 1D).

UGGT1 substrates were enriched for plasma membrane localized proteins (35\%) when compared to UGGT2 substrates (18\%), while plasma membrane proteins were found to compose a similar percent of the N-glycome (37\%) compared to UGGT1 substrates. Similar

11 percentages of UGGT1 and UGGT2 substrates localize to the ER (18\%), are secreted (12\%), or

12 are found in multiple localizations (6\%) (Figure 2C). Even though $4 \%$ of the $\mathrm{N}$-glycome is

13 composed of Golgi proteins (Figure 1D), neither UGGT1 nor UGGT2 appeared to modify Golgi

14 localized proteins.

The number of UGGT1 substrates was double that of UGGT2 suggesting that UGGT1

16 carried the main quality control load. Only three out of thirty-three UGGT2 substrates were

17 specific to UGGT2. These three UGGT2 specific substrates included arylsulfatase $A, \alpha-N-$

18 acetylgalactosaminidase and $\beta$-hexosaminidase subunit $\beta$ (HexB), three soluble lysosomal

19 enzymes (Figure 2D and E). Thirty substrates overlapped between UGGT1 and UGGT2, while

20 thirty-six substrates were found to be specific to UGGT1 (Figure 2D and Supplemental Table 3).

21 The preference for the shared substrates was explored by plotting all proteins identified as a

22 substrate of either glucosyltransferase on a $\log _{10}$ scale of the associated TMT value in

23 ALG6/UGGT2\% cells divided by the values in ALG6/UGGT1\% cells (Figure 2E). Proteins enriched

24 as UGGT2 substrates therefore possess positive values while UGGT1 enriched substrates have 25 negative values.

26 The three substrates found to be specific to UGGT2 clustered away from all other 27 proteins (Figure 2E at the top left). The remaining UGGT2 enriched substrates, except for one 28 ER localized protein, localized to the lysosome. All the UGGT2 favored substrates were soluble 29 proteins. In contrast, UGGT1 favored proteins were greater in number and displayed a diversity 
1 of localizations with a preference for plasma membrane proteins. These results indicate that

2 UGGT2 is a functional glucosyltransferase, which preferentially engages soluble lysosomal

3 proteins while UGGT1 modifies a wider variety of proteins with a preference for plasma

4 membrane and transmembrane domain-containing proteins in general.

5

6

7

8

9

10

11

12

13

14

15

16

17

18

19

20

21

22 


\section{Figure 2}

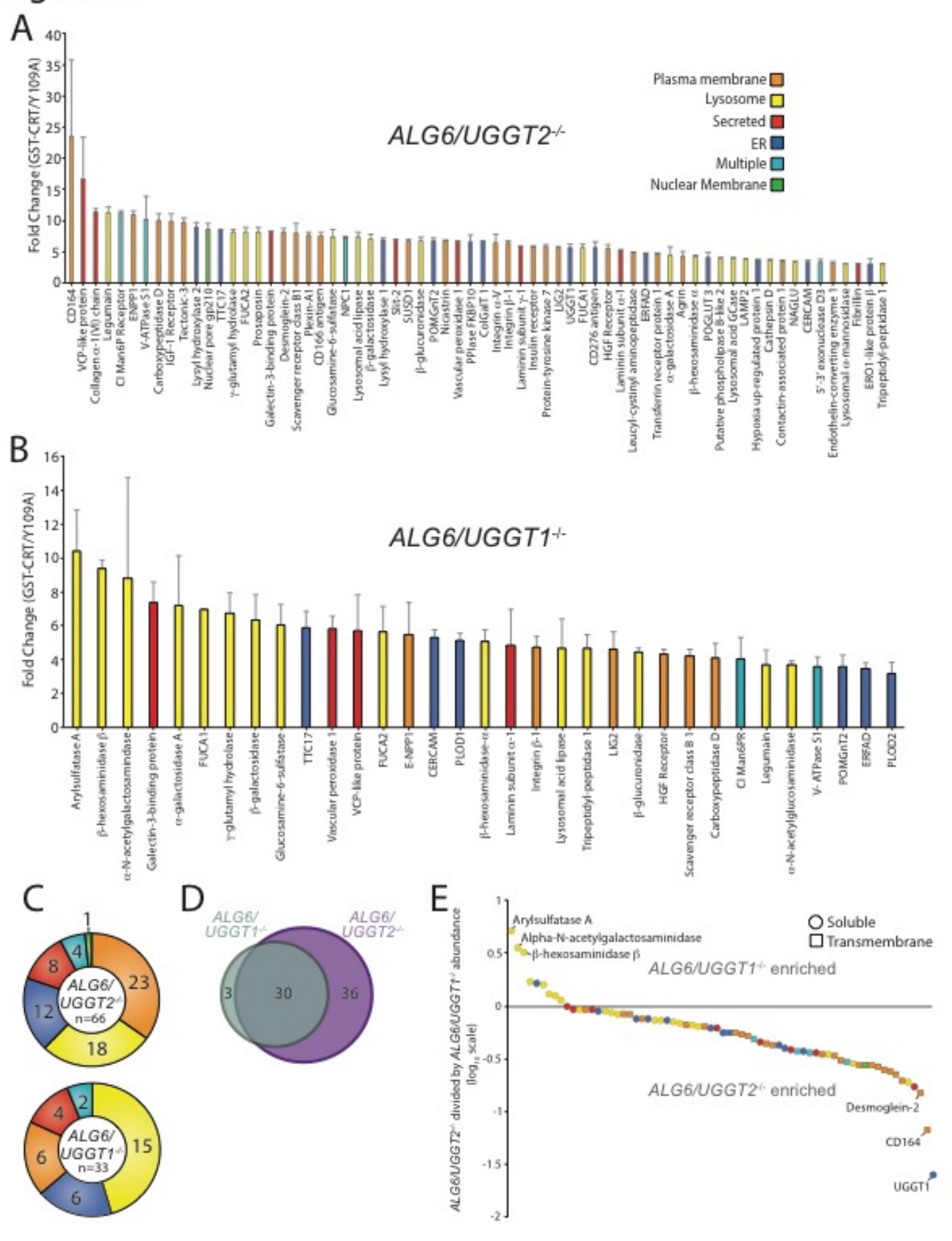

3 Figure 2. Identification of UGGT1 and UGGT2 specific substrates.

(A) Reglucosylation substrates in ALG6/UGGT2\% cells were identified and quantified as

5 previously described in Figure 1. Localizations as annotated by Uniprot are depicted. Data are 
1 representative of two independent experiments. Error bars represent SEM. (B) Reglucosylation

2 substrates in ALG6/UGGT1\% cells were identified and quantified as previously above. (C) The

3 distribution of localizations as annotated by Uniprot for reglucosylation substrates identified in

4 both $A L G 6 / U G G T 2 \%$ and $A L G 6 / U G G T 1 \%$ cells is depicted. (D) The overlap of reglucosylation

5 substrates identified in both $A L G 6 / U_{G G T 2}$ - cells (purple) and $A L G 6 / U G G T 1^{\%}$ cells (grey) is

6 visualized by a Venn diagram. (E) Reglucosylation substrate enrichment in either ALG6/UGGT1\%

7 or ALG6/UGGT2\% cells is depicted by dividing the TMT quantification for each protein in

8 ALG6/UGGT2\% cells by the associated value in ALG6/UGGT1\% cells on a $\log _{10}$ scale. Positive and

9 negative values represent enrichment in $A L G 6 / U G G T 1^{\%}$ and $A L G 6 / U G G T 2^{\%}$ cells, respectively.

10 Localization based on Uniprot annotation is depicted. Proteins are depicted as either circles

11 (soluble) or squares (transmembrane), as annotated by Uniprot.

12

13

14

15

16 


\section{Validation of UGGT substrates}

2 Having identified numerous novel substrates of the UGGTs, a select number of these substrates

3 were tested for reglucosylation to validate the identification approach. Substrates were chosen

4 based on a diversity of topologies, lengths, differences in propensities as UGGT1 or UGGT2

5 substrates and reagent availability. Monoglucosylated substrates were affinity isolated from ALG6\%, ALG6/UGGT1\%, ALG6/UGGT2\% and ALG6/UGGT1/UGGT2\% cells using GST-CRT compared to CST-CRT-Y109A. Substrates were then identified by immunoblotting with the percent reglucosylation determined by subtracting the amount of protein bound by GST-CRTY109A from that of GST-CRT, divided by the total amount of substrate present in the whole cell lysate, and multiplying by 100.

Cl-M6PR and insulin-like growth factor type 1 receptor (IGF-1R) are both large type I

12 membrane protein that possess multiple $\mathrm{N}$-glycosylation sites (Figure 3D and $\mathrm{H}$ ). Overall 10\% of

$13 \mathrm{Cl}$-M6PR was reglucosylated in $\mathrm{ALG6}^{\%}$ cells (Figure $3 \mathrm{~B}$ ). The modification level of $\mathrm{Cl}-\mathrm{M} 6 \mathrm{PR}$ was

14 significantly reduced in ALG6/UGGT1\%, but not ALG6/UGGT2\% cells. As a control,

15 reglucosylation was not observed in ALG6/UGGT1/UGGT2\% cells. A similar profile was observed

16 for IGF-1R where reglucosylation levels reached $12 \%$ in ALG6/UGGT2\%- cells (Figure 3E-G).

17 Altogether, these findings were consistent with the quantitative glycoproteomics isobaric

18 labeling results (Figure 3C and G), confirming that CI-M6PR and IGF-1R are efficient substrates 19 of UGGT1.

Next, the reglucosylation of the type II membrane protein, ectonucleotide

21 pyrophosphatase/phosphodiesterase family member 1 (ENPP1) was analyzed (Figure 3L).

22 ENPP1 was found to be reglucosylated at similar levels in $A L G 6^{-\%}(7 \%)$ and $A L G 6 / U G G T 1^{\%}(7 \%)$

23 cells. In ALG6/UGGT2\% cells, reglucosylation increased to $12 \%$, while in ALG6/UGGT1/UGGT2\%-

24 cells reglucosylation decreased to 1\% (Figure $3 \mathrm{I}$ and J). These results suggest that ENPP1 can be reglucosylated by both UGGT1 and UGGT2, with a slight preference for UGGT1, supporting the

26 TMT mass spectrometry results (Figure 3K).

27 The reglucosylation of the smaller soluble lysosomal protein, HexB, was also tested

28 (Figure 3M-P). HexB is processed into three disulfide-bonded chains in the lysosome (Mahuran

29 et al., 1988). Only immature or ER localized proHexB was affinity purified by GST-CRT (Figure 
$13 \mathrm{M}$, lanes 2, 5, 8 and 11 ). HexB was reglucosylated at $34 \%$ in $A L G 6^{-/}$cells (Figure $\left.3 \mathrm{~N}\right)$. No

2 significant change in glucosylation levels were observed when UGGT1 was also knocked out

3 (35\%). However, a reduction to $20 \%$ reglucosylation of HexB was observed in ALG6/UGGT2\%

4 cells, and complete loss of reglucosylation was observed in ALG6/UGGT1/UGGT2\% cells.

5 ALG6/UGGT1\% cells consistently displayed increased levels of expression of HexB (Figure 3M,

6 lane 4), which was supported by RNAseq data (Supplemental Figure 2B). These results confirm

7 the mass spectrometry results, which showed HexB to be a favored substrate of UGGT2 (Fig

8 30). It is also notable that HexB, as the first validated substrate of UGGT2, is highly

9 reglucosylated. Taken together, these results demonstrate that the mass spectrometry screen

10 accurately identified substrates of the UGGTs, as well as differentiated between substrates

11 specific to either UGGT1 or UGGT2.

12 


\section{Figure 3}
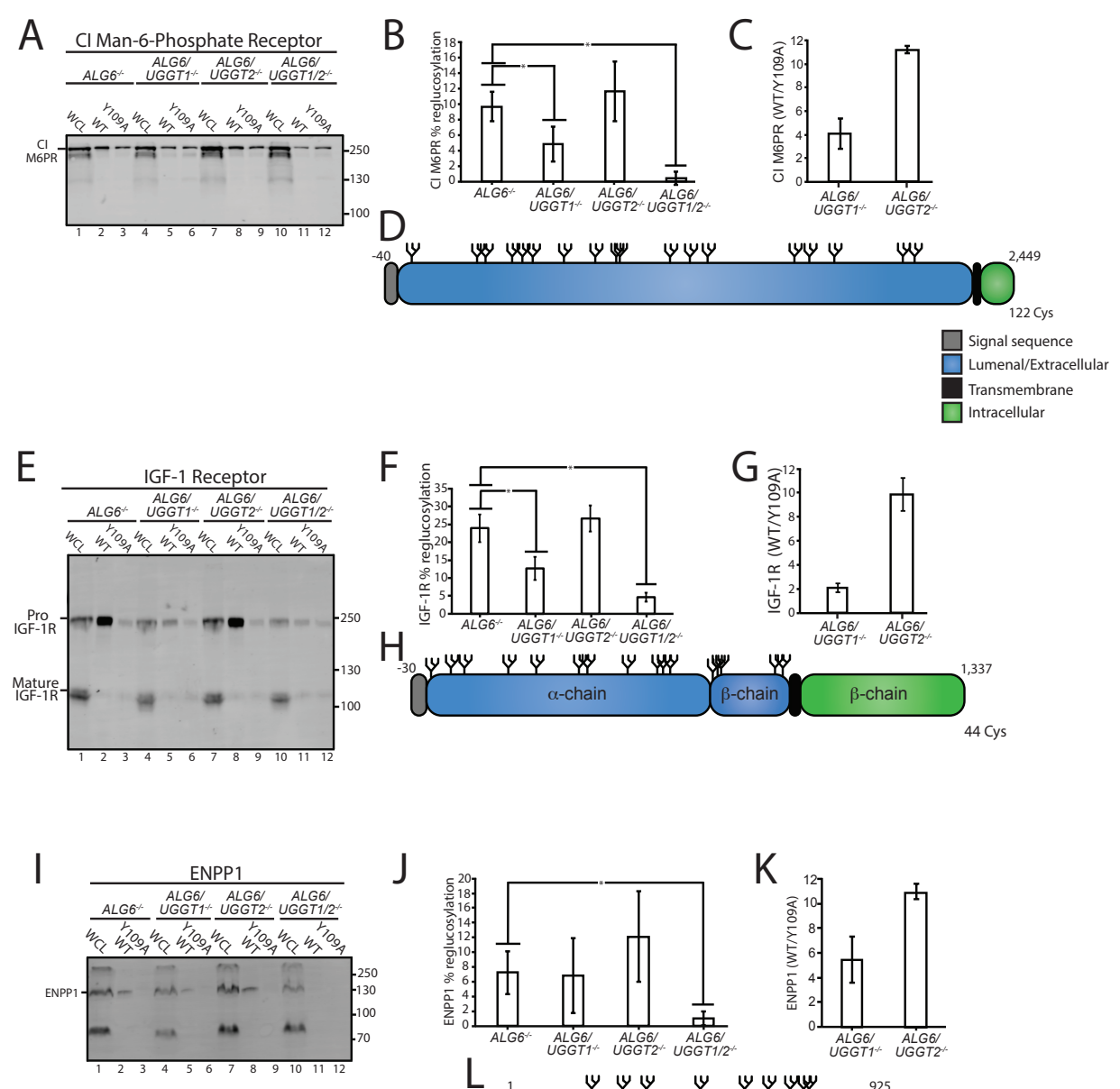

$\mathrm{L}$
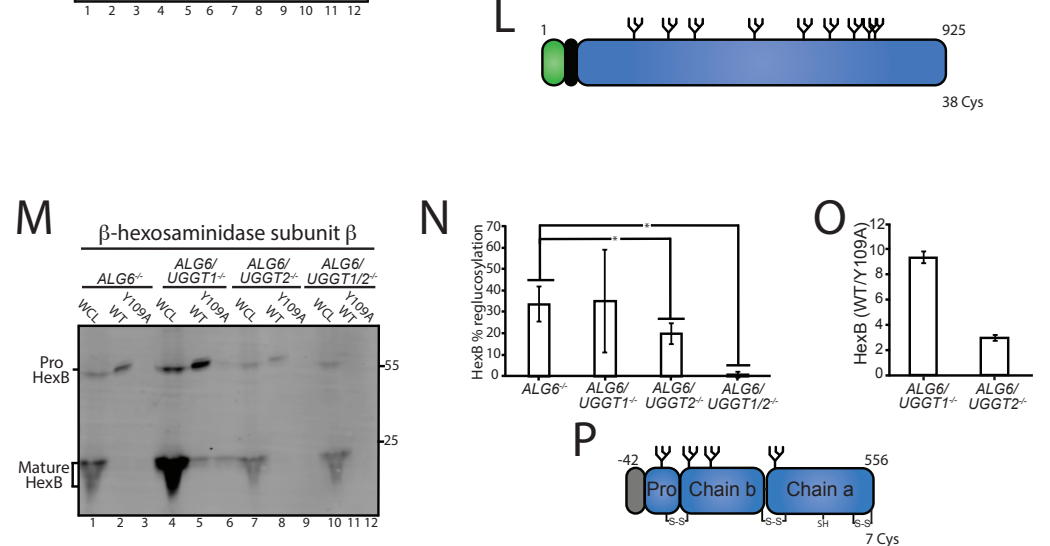

$P$

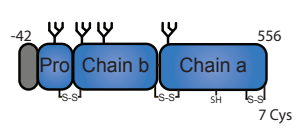

3 Figure 3. Validation of select reglucosylation substrates.

4 (A) The designated cell lines were lysed and split into whole cell lysate (WCL, 10\%) or affinity 5 purification by GST-CRT-WT or GST-CRT-Y109A and imaged by immunoblotting against the $\mathrm{Cl}$ 
1 Man-6-Phosphate receptor. Data is representative of three independent experiments with quantification shown in panel B. Quantifications were calculated by subtracting the value of

3 protein in the Y109A lane from the value of protein in the associated WT lane, divided by the

4 value of protein in the associated WCL lane. Error bars represent the standard deviation.

5 Asterisks denote a p-value of less than 0.05 (C) TMT mass spectrometry quantification of $\mathrm{Cl}$

6 Man-6-Phosphate receptor reglucosylation from ALG6/UGGT1\% cells (Figure 2B) and

7 ALG6/UGGT2\% cells (Figure 2A). (D) Cartoon representation of CI Man-6-Phosphate receptor

8 with $\mathrm{N}$-glycans (branched structures), the signal sequence (grey), luminal/extracellular domain

9 (blue), transmembrane domain (black) and intracellular domain (green) depicted. Number of

10 amino acids and Cys residues are indicated. (E) Reglucosylation of IGF-1R, conducted as

11 previously described above. Pro IGF-1R and mature IGF-1R are both observed due to proteolytic

12 processing. Data are representative of three independent experiments with quantification

13 displayed in F. (G) TMT mass spectrometry quantification of IGF-1R from Figure $2 A$ and $B$, as

14 previously described. (H) Cartoon depiction of IGF-1R. (I) The reglucosylation of ENPP1 shown

15 with quantification displayed in J. (K) TMT mass spectrometry quantification of ENPP1 from

16 Figure 2A and B with cartoon depiction of ENPP1 in L. (M) Reglucosylation of $\beta$-hexosaminidase

17 subunit $\beta$, conducted as previously described with quantifications displayed in N and TMT mass

18 spectrometry quantification of $\beta$-hexosaminidase subunit $\beta$ from Figure $2 A$ and $B$ in $O$ with a

19 cartoon depicting $\beta$-hexosaminidase subunit $\beta$ in $\mathrm{P}$. 


\section{Analysis of UGGT substrates}

2 To investigate the properties of the substrates modified by the UGGTs and identify potential

3 types of proteins UGGT1 and UGGT2 modify, a systematic analysis of the substrates of the

4 UGGTs was performed and compared to the general properties of the N-glycome. All

5 characteristics were analyzed using UniprotKB annotations. Initially, the length of substrates was compared to the $\mathrm{N}$-glycome. The $\mathrm{N}$-glycome ranged widely in size, from elabela (54 amino acids) to mucin-16 (14,507 amino acids). The overall amino acid distribution of the N-glycome was significantly shifted smaller compared to the size of UGGT substrates (Figure 4A). The median size of the $\mathrm{N}$-glycome was 443 amino acids, compared to 737 for UGGT substrates found in $A L G 6^{-/}$cells. Substrates of both UGGT1 (718 amino acid median) and UGGT2 (585

11 amino acids) are significantly larger when compared to the $\mathrm{N}$-glycome. This increase in length

12 may lead to more complex folding trajectories, requiring increased engagement with the lectin 13 chaperones for efficient maturation.

14 The distribution of the number of $\mathrm{N}$-glycans possessed by the $\mathrm{N}$-glycome (median of 2 15 glycans per glycoprotein) was also shifted significantly smaller than that of UGGT1 (7 glycans) or

16 UGGT2 (5 glycans) substrates (Figure 4B). All the UGGT substrates displayed both a larger 17 shifted peak and a prominent extended shoulder compared to the N-glycome. Despite the 18 identification of UGGT1 and UGGT2 substrates generally containing high numbers of N-glycans, 19 multiple substrates possessed as few as two N-glycans, suggesting that the experimental 20 approach did not require a high number of monoglucosylated glycans for GST-CRT affinity 21 isolation.

The ER maintains an oxidizing environment that supports the formation of disulfide

23 bonds. Complex folding pathways can involve the engagement of oxidoreductases, such as the 24 calnexin/calreticulin-associated oxidoreductase ERp57, to catalyze disulfide bond formation 25 and isomerization (Margittai and Sitia, 2011; Kozlov and Gehring, 2020). The most common 26 number of Cys residues in proteins identified as UGGT substrates was 2, which was similar to 27 the N-glycome Cys content (Figure 4C). However, there are variations in the median number of 28 Cys residues as for the $\mathrm{N}$-glycome it is 11 , which is smaller than that found in $A L G 6^{-/}$cells (16 Cys), and for UGGT1 substrates observed in ALG6/UGGT2\% cells (13 Cys). In contrast, a median 
1 of 9 Cys was observed for UGGT2 substrates. Therefore, UGGT1 appears to display a slight preference for proteins with high Cys content, when compared to the N-glycome and UGGT2 3 substrates.

UGGT1 or UGGT2 substrates displayed similar pl distributions with pls predominantly near a $\mathrm{pH}$ of 6.0 , while a second smaller peak centered around a $\mathrm{pH}$ of 8.5 . Interestingly, a pronounced valley was observed at $\mathrm{pH} 7.9$ under all conditions, presumably due to the instability of proteins with pls of a similar $\mathrm{pH}$ to that of the ER. The $\mathrm{N}$-glycome displayed a more bimodal distribution with significant population of both acidic and basic pls (Figure 4D). These results suggest that both UGGT1 and UGGT2 preferentially engage proteins with low pls.

The predicted topologies of the substrates of the UGGTs and the N-glycome were also

11 analyzed. Approximately $70 \%$ of the $\mathrm{N}$-glycome is comprised of membrane proteins, with half

12 of these membrane proteins possessing multiple transmembrane domains, followed by single

13 membrane pass proteins with a type I orientation (a third) with the remainder being type II

14 membrane proteins (Figure $4 \mathrm{E}$ ). A total of $43 \%$ of UGGT substrates in $A L G 6^{-/}$cells contained a transmembrane domain with the vast majority of these substrates having their C-terminus

16 localized to the cytosol in a type I orientation, while two substrates possessed the reverse type 17 II orientation and a single multi-pass membrane substrate (NPC1) was identified. When the 18 UGGTs were considered separately, about half of the UGGT1 substrates (ALG6/UGGT2\% cells) possessed at least one transmembrane domain, with $70 \%$ of these membrane proteins being in

20 the type I orientation, a quarter in a type II orientation and two being multi-pass proteins

21 (NPC1 and scavenger receptor class B member 1 (SR-BI)). In contrast to UGGT1, the majority of

22 UGGT2 substrates were soluble proteins (72\%) with the breakdown of remaining

23 transmembrane proteins being similar to that of UGGT1 with the majority being type I

24 membrane proteins. The preference of UGGTs for type I transmembrane proteins is likely caused by their larger luminal-exposed domains and $\mathrm{N}$-glycan numbers compared to multi-pass

26 membrane proteins (Figure 4F and G). Notably, substrates of the UGGTs had significantly larger

27 luminal domains than the membrane proteins of the $\mathrm{N}$-glycome, though especially for the 28 multi-pass membrane proteins (Figure 4F). Furthermore, while the pls of type II and polytopic membrane proteins were bimodal, they were overall more basic, which appears to be a 
1 property disfavored by UGGT substrates (Figure 4H). Overall, these results show that UGGT1

2 efficiently modifies both soluble and membrane associated proteins, while UGGT2 strongly

3 favors soluble substrates.

4

5

6

7

8

9

10

11

12

13

14

15

16

17

18

19

20

21

22

23

24

25

26

27

28

29 
Figure 4
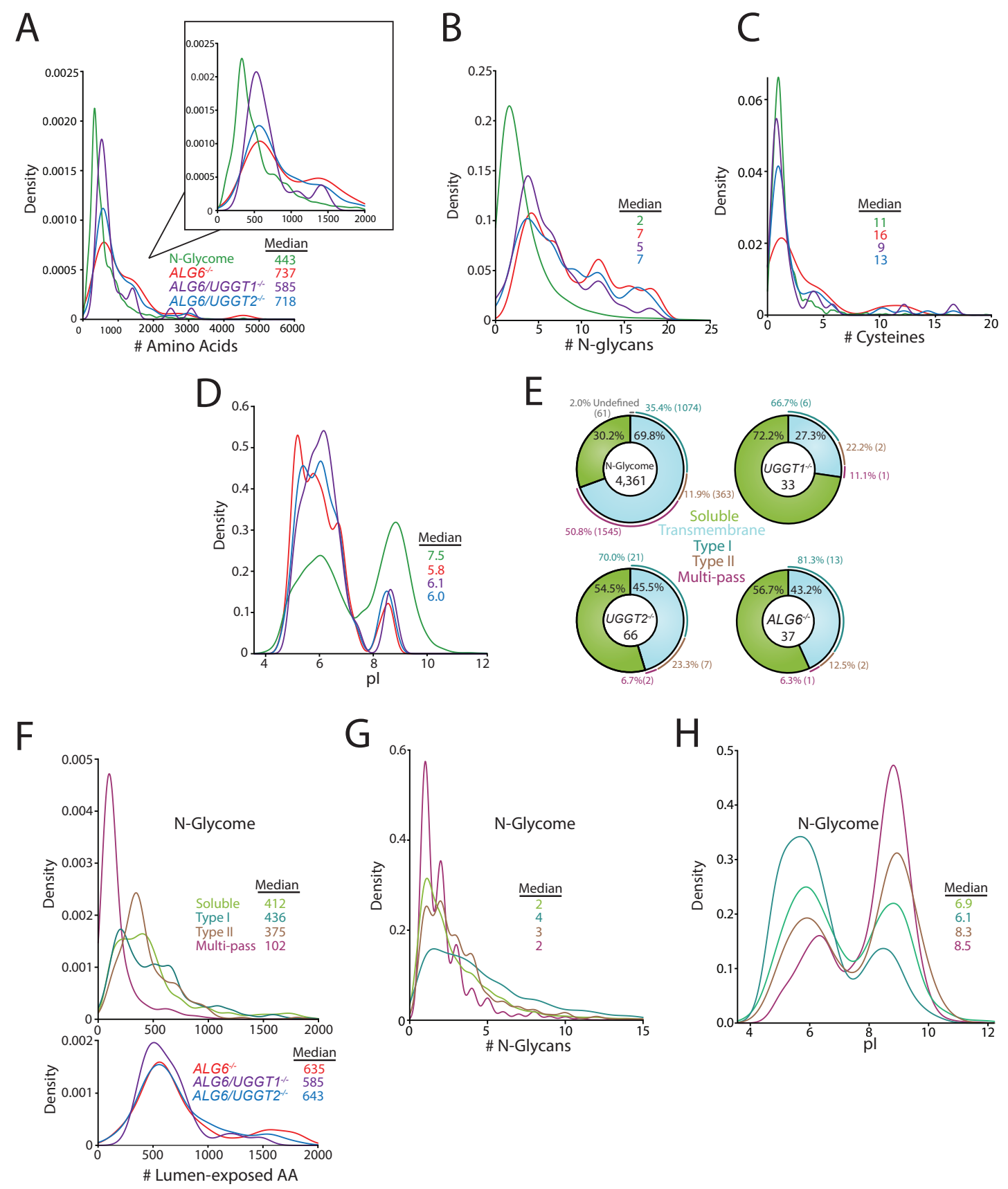

2 Figure 4. Analysis of substrates of the UGGTs and the N-glycome.

3 (A) Amino acid lengths of each protein in the indicated datasets was visualized by density plot,

4 with the total area under the curve integrated to 1 . Amino acid number was obtained via 
1 Uniprot annotation. All density plots were generated using R and the ggplot package. (B) The number of $\mathrm{N}$-glycans (B) or Cys residues (C) for each protein in the indicated datasets was

3 visualized by density plot with the numbers determined using their Uniprot annotation. (D) The

4 isoelectric point $(\mathrm{pl})$ values for each protein in the indicated datasets was visualized by density

5 plot. The pl values were obtained via ExPASy theoretical pl prediction. (E) The computationally

6 predicted $\mathrm{N}$-glycome and the indicated reglucosylation substrates were determined as either

7 soluble or transmembrane using Uniprot annotations. The transmembrane portion of each

8 dataset was then analyzed for type I, type II, or multi-pass topology using the associated

9 Uniprot annotation. Proteins which were annotated by Uniprot as transmembrane but lacked

10 topology information were labelled as undefined. (F) The computationally determined N-

11 glycome was separated into soluble, type I, type II, and multi-pass transmembrane proteins

12 using Uniprot annotations. Luminally exposed amino acids were computationally determined

13 using Uniprot annotations for each subset of the $\mathrm{N}$-glycome and each indicated reglucosylation

14 substrate dataset. The resulting data was visualized by density plot. (G) The indicated N-

15 glycome subsets were analyzed for $\mathrm{N}$-glycan content using Uniprot annotation and visualized by

16 density plot, as described. (H) The indicated N-glycome subsets were analyzed for predicted pl

17 using ExPASy theoretical pl prediction and visualized by density plot. 


\section{$1 \quad$ Efficient IGF-1R trafficking requires lectin chaperone engagement}

2 A number of natural substrates of the UGGTs were identified using a glycoproteomics approach

3 with gene edited cell lines. As reglucosylation by the UGGTs can direct multiple rounds of lectin

4 chaperone binding, the necessity for reglucosylation to support the efficient maturation of a

5 reglucosylated substrate was investigated. IGF-1R is proteolytically processed in the trans-Golgi

6 by proprotein convertases including furin, facilitating the monitoring of IGF-1R trafficking from

7 the ER to the Golgi (Lehmann et al., 1998). The requirement for lectin chaperone binding and

8 reglucosylation to aid IGF-1R trafficking was analyzed.

9 Initially, cells were treated without or with the inhibitor of $\alpha$-glucosidases I and II, DNJ,

10 to accumulate IGF-1R in the triglucosylated state to bypass entry into the calnexin/calreticulin

11 binding cycle (Helenius and Hammond, 1994; Hebert et al., 1995). At steady state as probed by

12 immunoblotting of cell lysates, IGF-1R accumulated in the ER localized pro form relative to the

13 mature form after DNJ treatment (Figure $5 \mathrm{~A}$ ), resulting in a $19 \%$ decrease in the level of the

14 trans-Golgi processed mature protein (Figure 5B). This indicated that the lectin chaperone

15 binding cycle helps support efficient IGF-1R trafficking.

16 There are two modes for engaging the lectin chaperone cycle: initial binding, which can

17 potentially commence co-translationally for glycoproteins such as IGF-1R that have N-glycans

18 located at their $\mathrm{N}$-terminus through their trimming of the terminal two glucoses by glucosidases

19 I and II; or by rebinding, which is directed by the reglucosylation of unglucosylated species by

20 the UGGTs (Parodi and Caramelo, 2015; Lamriben et al., 2016). The contribution of each mode

21 of monoglucose generation for the proper trafficking of IGF-1R was analyzed.

22 IGF-1R maturation was investigated in $\mathrm{ALG6}^{-}$cells as in these cells the N-glycan

23 transferred to the nascent substrate is non-glucosylated, leading to a lack of initial glucosidase

24 trimming mediated lectin chaperone binding. Reglucosylation by the UGGTs is required for

25 lectin chaperone binding in $A L G 6^{\%}$ cells. Similar to DNJ treatment in wild type cells, $A L G 6^{\%}$ cells

26 demonstrate a $20 \%$ decrease in mature IGF-1R relative to the pro form at steady state (Figure

27 5C, lanes 1 and 3, and Figure 5D). As hypoglycosylation can occur in a substrate dependent

28 manner in Alg6 ${ }^{-/}$cells (Shrimal and Gilmore, 2015), the mobility of IGF-1R with and without N-

29 glycans (PNGase F treated) was monitored by comparing the mobility of IGF-1R by SDS-PAGE 
1 and immunoblotting of wild type and $A L G 6^{-}$cell lysates. IGF-1R, and similarly, Cl-M6PR, appeared to be fully glycosylated, while HexB migrated faster when synthesized in $A L G 6^{-/}$cells likely due to hypoglycosylation (Figure 5C and Supplemental Figure 3C and D). To confirm that the pro form of IGF-1R represented ER localized protein rather than protein trafficked out of the ER but not processed by proprotein convertases, IGF-1R from wild-type and Alg6 ${ }^{-/}$cells was treated with the endoglycosidase EndoH. As EndoH cleaves high-mannose glycans which are preferentially present in the ER or early Golgi, an increase in mobility by SDS-PAGE suggests ER localization. In both wild-type and $A / g 6^{\%}$ cells, Pro IGF-1R was found to be EndoH sensitive, while mature IGF-1R was found to be largely EndoH resistant (Figure 5C, lanes 2 and 5), suggesting the accumulation of pro IGF-1R in Alg $6^{-/}$cells represents impaired ER trafficking

11 rather than impaired processing in the trans-Golgi. Altogether, these steady state results

12 suggest that lectin chaperone binding is important for efficient IGF-1R maturation.

As steady state results can be impacted by changes in protein synthesis and turnover, a

14 radioactive pulse-chase approach was used to follow protein synthesized during a $1 \mathrm{hr}\left[{ }^{35} \mathrm{~S}\right]-$

15 Met/Cys pulse interval followed by chasing for up to 2-hr under non-radioactive conditions.

16 Pulse-chase experiments are generally performed with overexpressed tag constructs to accumulate and isolate sufficient protein for monitoring. Here, endogenous IGF-1R was isolated by immunoprecipitation with anti-IGF-1R antibodies and analyzed by SDS-PAGE and autoradiography to determine the percent of IGF-1R that was properly processed to its mature

20 form in the trans-Golgi. IGF-1R was found to traffic efficiently out of the ER and to the Golgi in 21 wild type cells as $59 \%$ of the total protein after a 2 -hr chase was mature IGF-1R (Figure 5E, lanes 22 1-3 and F). When lectin chaperone binding was inhibited by treatment with DNJ, mature IGF-1R 23 was diminished to $22 \%$, underscoring the importance of lectin chaperone binding (Figure 5E, 24 lanes 4-6 and F).

To delineate the contributions of early compared to late lectin chaperone binding, IGF-

$261 \mathrm{R}$ trafficking was followed in gene edited cells that control the methods for lectin chaperone

27 engagement. A single early round of lectin chaperone binding will be permitted in the absence 28 of both UGGTs or rebinding would only be directed by the UGGT present with knockouts of a 29 single UGGT. Alternatively, early lectin chaperone binding as dictated by glucosidase trimming 
1 will be absent in the $A L G 6^{-/}$cells where lectin chaperone binding is directed solely through

2 glucosylation by the UGGTs. Monitoring the trafficking of IGF-1R in these cells will allow us to

3 determine the contributions of the different steps in the lectin chaperone binding cycle for

4 proper IGF-1R maturation.

5 When both UGGTs were absent in UGGT1/2\% cells, the percent of mature IGF-1R after 2

$6 \mathrm{hr}$ of chase decreased to $42 \%$. In agreement with early glycoproteomics and affinity isolation

7 results showing IGF-1R was largely a UGGT1 substrate, UGGT2 knockout alone had little

8 influence on IGF-1R trafficking while the knocking out of UGGT1 supported IGF-1R trafficking

9 similar to the double UGGT deletion (Figure 5E, 7-15 lanes and F). These results support a role

10 for UGGT1 in optimizing IGR-1R trafficking.

11 To determine the importance of early chaperone binding directed by the glucosidases,

12 IGF-1R trafficking was monitored in $\mathrm{ALG6}^{-/}$cells that support reglucosylation but lack the ability

13 for early binding to the lectin chaperones as directed by glucosidase trimming of the

14 triglucosylated species. In $A L G 6^{-}$cells, the percent of mature IGF-1R was significantly

15 decreased to $21 \%$, indicative of an important contribution of the initial round of lectin binding,

16 as was suggested by steady state data (Fig $5 C$ ). The addition of DNJ to $A L G 6^{-/}$cells would be

17 expected to trap IGF-1R in a monoglucosylated state after glucosylation, allowing the effect of

18 prolonged interaction with the lectin chaperones to be observed. Under this condition, IGF-1R

19 was strongly retained in the ER with no increase observed in the level of mature IGF-1R

20 observed even after $2 \mathrm{hr}$ of chase (Figure 5E, lanes 16-21 and F). Altogether these results

21 demonstrate that while early (glucosidase-mediated) and late (UGGT-mediated) lectin

22 chaperone binding contribute to the efficient trafficking from the ER and subsequent Golgi

23 processing of IGF-1R, early lectin chaperone binding appears to be most critical for supporting

24 proper IGF-1R maturation. 


\section{Figure 5}
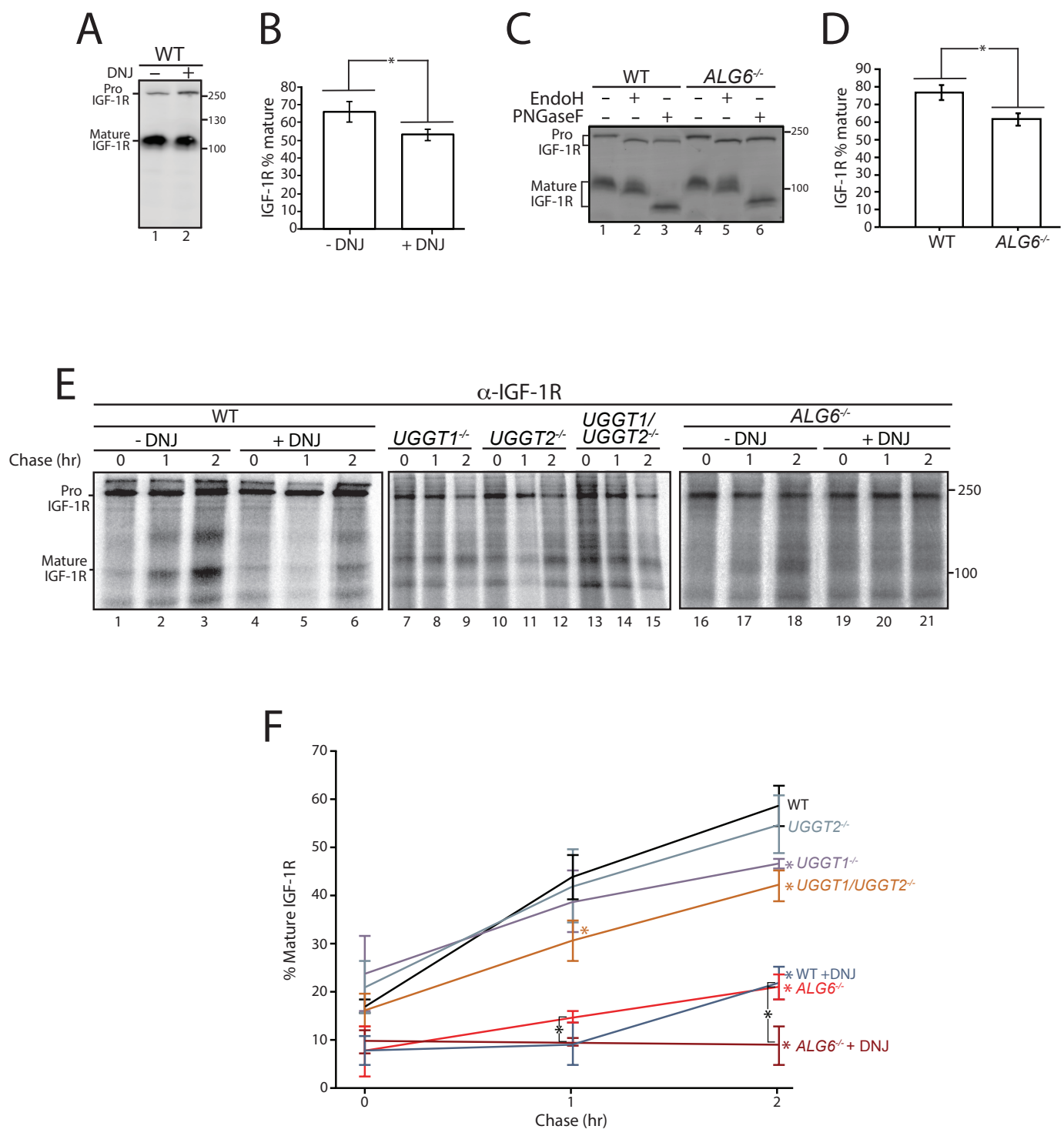

2 Figure 5. Calnexin/calreticulin cycle role for IGF-1R trafficking.

3 (A) WT HEK293-EBNA1-6E cells treated without or with DNJ $(500 \mu \mathrm{M})$ for 12-hr were lysed and

4 WCL samples were resolved by reducing 9\% SDS-PAGE and imaged by immunoblotting against 
$1 \quad$ IGF-1R. Data are representative of three independent experiments with quantification shown in

2 B. Percent of IGF-1R mature was calculated by dividing the amount of mature protein by the

3 total protein in each lane. Errors bars represent standard deviation. Asterisk denotes a p-value

4 of less than 0.05 (C) The indicated cell lines were lysed in RIPA buffer. Samples were split evenly

5 between non-treated and PNGaseF or EndoH treated. Samples were visualized by

6 immunoblotting against IGF-1R and data are representative of three independent experiments

7 with quantification displayed in D. (E) Indicated cells were treated without or with DNJ, pulsed

8 with $\left[{ }^{35} \mathrm{~S}\right]-$ Met/Cys for $1-\mathrm{hr}$ and chased for the indicated times. Cells were lysed and samples

9 were immunoprecipitated using anti- $\beta$ IGF-1R antibody and resolved by reducing SDS-PAGE and

10 imaged by autoradiography. Data are representative of three independent experiments with

11 quantification shown in $\mathrm{F}$

12

13

14

15

16 


\section{Figure 6}
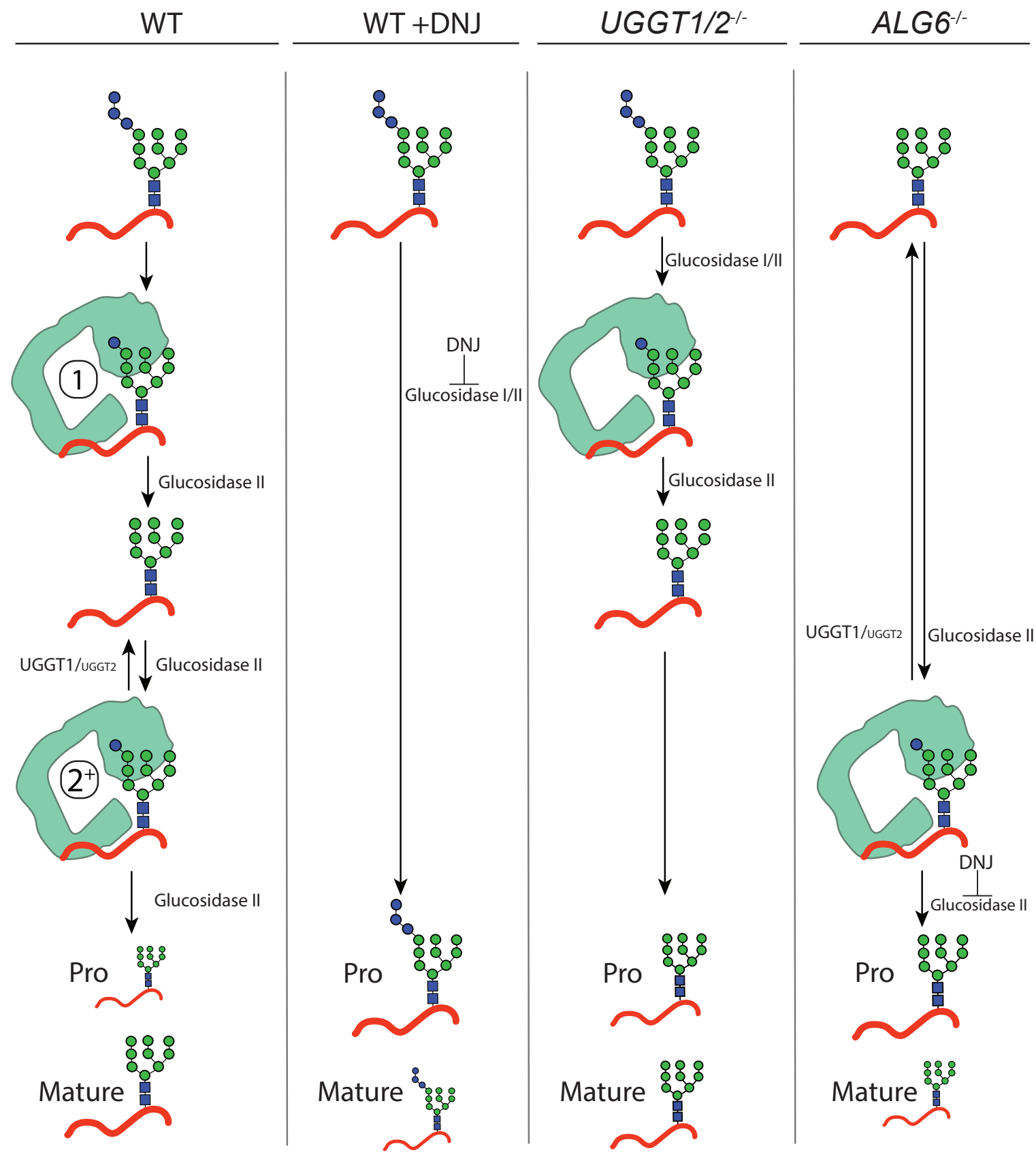

Figure 6. Model for IGF-1R engagement by the lectin chaperone cycle.

3 In WT cells, N-glycans with three terminal glucoses are appended to IGF-1R. Trimming of two

4 terminal glucoses by glucosidases I/II generates a monoglucosylated protein which supports an 
1 initial round of interaction with calreticulin (calnexin not shown, denoted by a 1). Trimming of

2 the final glucose by glucosidase II yields a non-glucosylated N-glycan. If recognized as non-

3 native primarily by UGGT1, and to a lesser extent UGGT2, IGF-1R may then be reglucosylated,

4 supporting a second round of interaction with calreticulin (denoted by a $2^{+}$). Multiple rounds of

5 trimming, reglucosylation and binding to calnexin or calreticulin can occur until proper folding

6 and trafficking. Under this system, IGF-1R is efficiently trafficked from the ER and mature IGF-

7 1R accumulates. When glucosidase I/II activity is inhibited by treatment with DNJ in WT cells, all

8 rounds of binding to the lectin chaperones are ablated and IGF-1R is retained in the ER, yielding

9 primarily pro IGF-1R. In UGGT1/2\% cells, initial binding to calnexin or calreticulin directed by

10 glucosidases $\mathrm{I} / \mathrm{II}$ trimming is maintained but rebinding via reglucosylation does not occur. Under

11 this system, IGF-1R is inefficiently trafficked from the ER. In ALG6-/- cells, N-glycans are

12 transferred without glucoses, eliminating the initial round of binding to calnexin or calreticulin

13 by glucosidases trimming. Only the second round of binding is supported by UGGT1, and to a

14 lesser extend UGGT2, mediated reglucosylation. Upon treatment with DNJ, reglucosylated IGF-

$151 \mathrm{R}$ may persistently interact with the lectin chaperones resulting in ER retention. 


\section{DISCUSSION}

As lectin chaperone binding is directed by the covalent modification of substrates by the UGGTs, the identification of bona fide substrates of the UGGTs is central to understand the impact the lectin chaperone network has on cellular homeostasis. Features of proteins alone cannot accurately predict which chaperones will be required for efficient folding and quality control (Adams et al., 2019b). Previous studies involving the UGGTs have focused mainly on the overexpression of biasedly selected substrates or using purified proteins, providing uncertain biological relevance (Ritter and Helenius, 2000; Taylor et al., 2003; Caramelo et al., 2004; Soldà et al., 2007; Pearse et al., 2008; Ferris et al., 2013; Tannous et al., 2015). Here, we used a quantitative glycoproteomics-based strategy to identify seventy-one natural cellular substrates of the UGGTs. When compared to the $\mathrm{N}$-glycome that represents the total population of potential substrates (4,361 N-glycoproteins in human cells), the UGGTs favored the modification of more complex, multidomain proteins with large numbers of $\mathrm{N}$-glycans. These results are in agreement with the common requirement of chaperones for the proper folding of more complex proteins (Balchin et al., 2016, 2020). The lectin chaperone system is part of the robust chaperone network necessary to promote the efficient folding and quality control of substrates and mitigate harmful misfolding events that are associated with a large range of pathologies.

The discovery of 33 UGGT2 cellular substrates provides the first evidence of intact UGGT2 acting as a quality control factor in cells (Figure 2B). Previous work demonstrated that UGGT2 is enzymatically active against chemically engineered glycosylated substrates using purified components or when the catalytic domain of UGGT2 was appended to the folding sensor domain of UGGT1 (Arnold and Kaufman, 2003; Takeda et al., 2014). The lower number of UGGT2 substrates compared to UGGT1 (66 substrates) is likely due, at least in part, to UGGT2 being expressed at a fraction of the level of UGGT1 ( $4 \%$ in HeLa cells (Itzhak et al., 2016)). Of special note is the preference of UGGT2 for lysosomal substrates as 8 of the 9 preferential UGGT2 substrates are lysosomal proteins (Figure 2E). The preferential UGGT2

28 substrates are all soluble proteins, while half of the preferential UGGT1 substrates contained transmembrane domains indicative of a further preference of UGGT2 for soluble proteins 
1 (Figure 2E). Given the preference of UGGT2 for soluble lysosomal proteins, it would be of interest in future studies to examine lysosomes in UGGT2\% cells as a number of the UGGT2 substrates are associated with lysosomal storage diseases including metachromatic leukodystrophy (arylsulfatase A), Sandhoff disease ( $\beta$-hexosaminidase subunit $\beta$ ) and Schindler disease ( $\alpha$-N-acetylgalactosaminidase) (Mahuran, 1999; Cesani et al., 2016; Ferreira and Gahl, 2017).

UGGT1 serves as the predominant ER glycoprotein quality control sensor. While overall the 66 UGGT1 substrates are evenly distributed between soluble and membrane proteins, the majority of the most efficiently reglucosylated proteins are membrane proteins (Figure 2E). Seventy percent of the membrane proteins modified by UGGT1 are in the type I orientation possessing luminal $\mathrm{N}$-glycosylated domains of significant length. Only two substrates of the UGGTs are multi-pass membrane proteins (NPC1 and SR-BI). In contrast to most polytopic membrane proteins that have little exposure to the ER lumen (Figure 4F), both NPC1 and SR-BI

14 have large heavily glycosylated luminal domains. The enrichment of UGGT1 for transmembrane 15 proteins may be influenced through a weak association with the ER membrane or a general

16 slower and more complex folding process for membrane proteins that provides a longer 17 window for modification.

18 An important question to ask is what is the basis for the differing substrate specificities of UGGT1 and UGGT2? They display sequence identities that are high within the catalytic

20 domains (83\% identical) and lower in their folding sensor domains (49\%) (Arnold and Kaufman,

21 2003). This sequence disparity within the folding sensor domain may drive altered substrate

22 selection. In addition, UGGT1 and UGGT2 may reside in separate subdomains within the ER, 23 which could contribute to substrate accessibility. The CLN6/CLN8 transmembrane complex 24 appears to recognize lysosomal proteins within the ER for COPII packaging in support of a 25 possible mechanism of lysosomal substrate selection (Bajaj et al., 2020). An additional 26 possibility addressed was that the level of expression of the lysosomal proteins identified as 27 UGGT2 substrates may be augmented in ALG6/UGGT1\%cells. However, only the mRNA expression level of $\beta$-hexosaminidase subunit $\beta$ was increased relative to $A L G 6^{\%}$ or wild type 29 cells, as supported by immunoblot data (Figure 3M), with the remaining preferential UGGT2 
1 lysosomal substrates displaying no significant change in mRNA expression levels (Supplemental

2 Figure 4). The increased expression of $\beta$-hexosaminidase subunit $\beta$ in ALG6/UGGT1\% cells may

3 be attributed to induction by UPR, as in these cells a slight induction primarily through the ATF6

4 branch of the UPR was observed (Supplemental Figure 5). Further studies will be required to

5 understand the varying selectivities of the UGGTs.

With some 4,350 possible N-glycosylated proteins as potential UGGT substrates, why were only 71 proteins identified as substrates of the UGGTs? First, many proteins are expected to fold in a chaperone independent manner, especially small, simple proteins. Second, our stringent isolation approach prioritized high quality substrates with at least a 3-fold induction for GST-CRT/GST-CRT-Y109A binding. Third, the profile of reglucosylated substrates is likely cell-

11 type dependent with additional substrates expected to be identified in cell types with heavy

12 secretory pathway loads such as pancreatic cells or hepatocytes, compared to the kidney line 13 used here. Fourth, 1,500 proteins of the $\mathrm{N}$-glycome are multi-pass transmembrane proteins

14 (Figure 4E). This class of protein was strongly de-enriched as substrates of the UGGTs, likely due to their limited luminal exposure and minimal N-glycan content (Figure 4F and G). This reduces the pool of favored substrates by a third. Fifth, the monoglucosylated protein isolation

17 procedure may also be limited by possibly requiring multiple sites of reglucosylation for

18 efficient binding to survive the pulldown protocol. However, multiple substrates with two N-

19 glycans were identified, suggesting heavy glycosylation is not an absolute requirement.

20 Additionally, protein expression levels are expected to play some role in substrate identification

21 but it does not appear to be a major determining factor as multiple strong substrates were

22 expressed at or below an average protein level for the $\mathrm{N}$-glycome and no correlation between

23 mRNA expression level and the TMT mass spectrometry fold increase for the GST-CRT/GST-CRT-

24 Y109A fraction was observed (Supplemental Figure 2). It would be of interest to determine if

25 proteotoxic stress would increase levels and the range of reglucosylated substrates as both the

26 pool of non-native proteins and the amount of the UPR-induced substrates of the UGGTs would

27 be expected to increase. It is also possible that some proteins identified as substrates of the

28 UGGTs may misfold after missing the first round of calnexin/calreticulin binding in $A L G 6^{\%}$ cells

29 and therefore engage the UGGTs more efficiently. 
As carbohydrate binding can be dictated initially by glucosidase trimming followed by additional later rounds of binding dictated by UGGT reglucosylation, it is of importance to understand which stage of the binding cycle contributes most significantly to proper protein maturation and cell homeostasis. N-glycans in Sacchromyces cerevisiae and other single cell species are transferred post-translationally as they are missing the OST isoform subunit that interacts with the Sec61 translocon and supports early co-translational modification (RuizCanada et al., 2009; Shrimal et al., 2019). A second OST isoform appears in multicellular organisms that is translocon-associated. In addition, reglucosylation activity was first observed in single cell parasites of Trypanosoma cruzi where glycans are transferred as Man, $\mathrm{GlcNAc}_{2}$ moieties thereby bypassing the initial glucosidase initiated binding step observed in metazoans

11 (Parodi and Cazzulo, 1982). These seminal T. cruzi studies from Parodi and colleagues that first 12 discovered the (re)glucosylation activity, later attributed to UGGT1, were the inspiration for the 13 development of the experimental $A L G 6^{-/}$system used in this study to isolate substrates of the 14 UGGTs. Conservation analysis of glycosylation and the lectin chaperone pathway suggests that reglucosylation supporting the quality control function of the calnexin cycle evolved prior to its role in assisting in earlier folding events.

Using CRISPR edited cell lines, the contributions of the various steps for chaperone

18 binding engagement for the UGGT1 substrate IGF-1R was experimentally explored as its processing in the Golgi provided a robust Golgi trafficking assay. Furthermore, IGF-1R is a target

20 in cancer biology as it is important for cell growth (Sell et al., 1994; Desbois-Mouthon et al.,

21 2006; Chng et al., 2006; King et al., 2014; Mutgan et al., 2018). When binding to the lectin

22 chaperones was blocked in wild type cells by glucosidase inhibition with DNJ treatment,

23 supporting the production of triglucosylated trapped species, the percent of processed IGF-1R

24 strongly decreased compared to untreated cells, demonstrating a requirement of lectin

25 chaperone engagement for the efficient maturation, trafficking and processing of IGF-1R. In

$26 U G G T 1 / 2^{\%}$ cells, IGF-1R can enter the first round of glucosidase-mediated binding to the lectin

27 chaperones but rebinding directed primarily by UGGT1 mediated reglucosylation cannot occur

28 (Figures 5E, F and 6). This led to a reduced efficiency in the accumulation of mature IGF-1R. The

29 first round of lectin chaperone binding is bypassed in $A L G 6^{\%}$ cells as the N-glycans transferred 
1 to proteins do not contain glucoses (Figure 6). Therefore, only the rebinding events mediated

2 by reglucosylation take place. More strikingly in $A L G 6^{-/}$cells, this led to a dramatic reduction in

3 IGF-1R processing at a greater level than in UGGT1/2\%- cells, indicating the first round of binding

4 to the lectin chaperones was most critical for IGF-1R maturation. The addition of DNJ in ALG6-1-

5 cells supported the trapping of reglucosylated side chains and severely reduced Golgi

6 processing, suggesting that reglucosylation-mediated persistent interaction with the lectin

7 chaperones delays IGF-1R exit from the ER.

Understanding the proteins that interact with or rely on chaperone systems will advance

9 our understanding of protein homeostasis (Houry et al., 1999; Kerner et al., 2005). Large multi-

10 domain proteins such as IGF-1R and many of the other substrates of the UGGTs have

11 apparently evolved to utilize the lectin chaperone system to help direct their complex folding

12 trajectories. The co-evolution of chaperones and their substrates has led to the expansion of

13 the complexity of the proteome for multicellular organisms (Balchin et al., 2016; Rebeaud et al.,

14 2020). The large group of substrates of the UGGTs identified here represents glycoproteins that

15 utilize multiple rounds of lectin chaperone engagement for proper maturation and are likely

16 more prone to misfold under stress. Future studies will determine if this increased vulnerability

17 makes these substrates more susceptible to misfold under disease conditions where cell

18 homeostasis is challenged.

20 EXPERIMENTAL METHODS

21 Reagents

22 Antibodies used were: rabbit monoclonal IGF-1 receptor $\beta$ (D23H3, Cell Signaling), rabbit

23 monoclonal IGF-IIR/CI-M6PR (D3V8C, Cell signaling), rabbit monoclonal BiP (C50B12, Cell

24 Signaling), rabbit monoclonal $\beta$-hexosaminidase subunit $\beta$ (HEXB) (EPR7978, Abcam), rabbit

25 polyclonal ENPP1 (N2C2, Genetex) rabbit polyclonal UGGT1 (GTX66459, Genetex), mouse

26 monoclonal Glyceraldehyde 3-Phosphate (MAB374, Millipore Sigma), IRDye x anti-rabbit

27 secondary (LiCor). All chemicals were purchased from Millipore-Sigma, except where indicated. 
1 HEK293-EBNA1-6E cells were employed and used as the parental line to create all CRISPR/Cas9 edited lines (Tom et al., 2008). Cells were cultured in DMEM (Sigma) supplemented with certified $10 \%$ fetal bovine serum (Gibco) at $37{ }^{\circ} \mathrm{C}$ at $5 \% \mathrm{CO}_{2}$. Cells were tested for the presence of mycoplasma using a universal mycoplasma detection kit (ATCC, Cat \# 30-012K).

\section{CRISPR/Cas9-mediated knock outs}

HEK293EBNA1-6E ALG6\% , ALG6/UGGT1\%, ALG6/UGGT2\%, ALG6/UGGT1/UGGT2\%, UGGT1\%,

UGGT2\%, and UGGT1/2\% cells were generated via CRISPR/Cas9 using gRNA plasmids gh260, gh172, and gh173, and Cas9-GFP plasmid CAS9PBKS (Lonowski et al., 2017; Narimatsu et al., 2018). Plasmids gh260 (106851), gh172 (106833), gh173 (106834), and CAS9PBKS (68371) were

11 from Addgene. Knock-out cell lines were generated by co-transfecting HEK293-EBNA1-6E cells

12 at $70 \%$ confluency in a $10-\mathrm{cm}$ plate with $7 \mu \mathrm{g}$ of both the associated gRNA and Cas9-GFP

13 plasmid, using a $2.5 \mu \mathrm{g}$ of PEI per $1 \mu \mathrm{g}$ of plasmid. Cells were allowed to grow for $48 \mathrm{hr}$ prior to

14 trypsinization and collection. After trypsinization, cells were collected and washed twice with

15 sorting buffer (1\% FBS, 1mM EDTA, PBS). Cells were then resuspended in sorting buffer at

16 approximately 1 million cells per $\mathrm{ml}$. Cells were then bulk separated using flow assisted cell

17 sorting based on the top $10 \%$ of Cas9-GFP expressing cells (FACS Aria II SORP, Becton Dickinson

18 and Company). Cells were then plated at 5, 10, 20 thousand cells per $10 \mathrm{~cm}$ plate in pre-

19 conditioned DMEM media with $20 \%$ FBS. Colonies derived from a single cell were isolated using

20 cell cloning cylinders (Bellco Glass), trypsinized from the plate, and further passaged. Knock-

21 outs were confirmed by immunoblotting and staining for UGGT1 or, where antibodies were not

22 available, isolating genomic DNA using a genomic DNA isolation kit (PureLink genomic DNA mini

23 kit, Thermo Fisher), PCR amplification of the genomic DNA region of interest, and insertion of

24 genomic DNA into pcDNA3.1-. Plasmids were then sequenced for conformation (Genewiz).

\section{GST-CRT purification}

27 The plasmid for pGEX-3X GST-CRT was from Prof. M. Michalak (University of Alberta). pGEX-3X GST-calreticulin-Y109A was generated by site-directed mutagenesis. GST-CRT was expressed in 
1 with shaking until an O.D. of $A_{600}=0.6$. Protein expression was then induced by treating cultures with $8.32 \mathrm{mg} / \mathrm{L} \mathrm{IPTG}$ for $2 \mathrm{hr}$. Cultures were centrifuged at 3,000 g for $10 \mathrm{~min}$. Cell pellets were

3 lysed with cold lysis buffer (1 mM phenylmethylsulfonyl fluoride, 2\% Triton X-100, PBS pH 7.4) and resuspended. Resuspended cells were lysed in a microfluidizer (110L, Microfluidics) at $18,000 \mathrm{psi}$ for two passes. The cell lysate was centrifuged for $40 \mathrm{~min}$ at $8,000 \mathrm{~g}$ at $4{ }^{\circ} \mathrm{C}$. Lysate was filtered through a $0.45 \mu \mathrm{m}$ filter. Two $\mathrm{ml}$ bed volume glutathione sepharose beads (GE Lifesciences, Cat\# GE17-0756-01) per liter of lysate was equilibrated in wash buffer (1\% Triton $\mathrm{X}-100,1 \mathrm{mM}$ PMSF, PBS pH 7.4), added to cleared lysate, and rotated at $4{ }^{\circ} \mathrm{C}$ for $3 \mathrm{hr}$. Beads were precipitated through centrifugation at $1,000 \mathrm{~g}$ for $5 \mathrm{~min}$ at $4{ }^{\circ} \mathrm{C}$. The supernatant was aspirated and beads were washed twice in wash buffer with gentle resuspension between washes. One $\mathrm{ml}$ of elution buffer (10 $\mathrm{mM}$ reduced glutathione, $1 \mathrm{mM}$ PMSF, $50 \mathrm{mM}$ Tris pH 8.5) was added to beads and beads were gently resuspended and allowed to incubate for 5 min at 4 ${ }^{\circ} \mathrm{C}$. Beads were precipitated by centrifugation at $1,000 \mathrm{~g}$ for $5 \min 4{ }^{\circ} \mathrm{C}$. The eluate was collected and a total of 6-elutions were collected. Resulting eluate was tested for purity and protein amount on a reducing SDS-PAGE and stained with Imperial protein stain (Thermo Fisher, Cat\#

16 24617). Elutions were then combined and protein concentration was quantified by a Bradford

17 assay (Bio-Rad). Purified protein was then stored at $-80{ }^{\circ} \mathrm{C}$ in a $20 \%$ glycerol PBS buffer at 1

$18 \mathrm{mg} / \mathrm{ml}$.

\section{GST-CRT isolation and TMT mass spectrometry sample preparation}

21 Five $10 \mathrm{~cm}$ plates were seeded with 3.5 million cells and allowed to grow for $48 \mathrm{hr}$. Cells were 22 treated with N-butyldeoxynojirimycin hydrochloride (DNJ) Cayman Chemicals, Cat \# 21065) at $23500 \mu \mathrm{M}$ for $1 \mathrm{hr}$. Prior to lysis, the media was aspirated and cells were washed once with filter 24 sterilized PBS. Cells were lysed in $1 \mathrm{ml}$ of lysis buffer $(20 \mathrm{mM} \mathrm{MES}, 100 \mathrm{mM} \mathrm{NaCl}, 30 \mathrm{mM}$ Tris pH

$257.5,0.5 \%$ Triton X-100) per plate. Samples were shaken at $4{ }^{\circ} \mathrm{C}$ for $5 \mathrm{~min}$ and centrifuged at $2620,800 \mathrm{~g}$ at $4{ }^{\circ} \mathrm{C}$ for $5 \mathrm{~min}$. Lysate was pre-cleared with $25 \mu \mathrm{l}$ bed volume of buffer-equilibrated 27 glutathione beads per $1 \mathrm{ml}$ of lysate under rotation for $1 \mathrm{hr}$ at $25 \mu \mathrm{l}$ bed volume. Beads were 28 precipitated by centrifugation at $950 \mathrm{~g}$ at $4{ }^{\circ} \mathrm{C}$ for $5 \mathrm{~min}$. Glutathione beads were pre-incubated 29 with either GST-CRT or GST-CRT-Y109A by equilibrating $25 \mu \mathrm{l}$ bed volume/pull-down 
1 glutathione beads with lysis buffer. Beads were incubated with $100 \mu \mathrm{g}$ of purified GST-CRT/pull-

2 down under gentle rotation at $4{ }^{\circ} \mathrm{C}$ for $3 \mathrm{hr}$. Beads were centrifuged at $950 \mathrm{~g}$ at $4{ }^{\circ} \mathrm{C}$ for $5 \mathrm{~min}$

3 and washed twice with lysis buffer. Supernatant was collected and split in half, with one half

4 incubated for $14 \mathrm{hr}$ at $4{ }^{\circ} \mathrm{C}$ under gentle rotation with glutathione beads pre-incubated with

5 GST-CRT and the other half under the same conditions with GST-CRT-Y109A.

After incubation with GST-CRT beads, samples were washed once in lysis buffer without protease inhibitors and twice in $100 \mathrm{mM}$ triethylammonium bicarbonate (Thermo Fisher Cat\# 90114). After the final wash, samples were incubated with $10 \mu \mathrm{l}$ of $50 \mathrm{mM}$ DTT (Pierce, Cat\# A39255) for $1 \mathrm{hr}$ at room temperature under gentle agitation. Samples were treated with $2 \mu \mathrm{l}$ of $125 \mathrm{mM}$ iodoacetamide (Pierce, Cat\# A39271) and incubated for 20 min under gentle

11 agitation, protected from light. Samples were digested with $5 \mu \mathrm{g}$ of trypsin (Promega, Cat\#

12 V5280) at $37^{\circ} \mathrm{C}$ overnight under agitation. Peptide concentration was quantified using a BCA

13 protein quantification kit (Pierce, Cat\# 23227). 10plex tandem mass tags (TMT) (Themo Fisher

$140.8 \mathrm{mg}$ ) were resuspended in mass spectrometry grade acetonitrile and was added to digested

15 peptide and incubated for 1-hr at room temp, per manufacturer's instructions. Labeling was

16 quenched by adding hydroxylamine to $0.25 \%$ and incubating for $15 \mathrm{~min}$ at room temp. Labeled

17 samples were pooled, treated with 1,000 units of glycerol-free PNGaseF (NEB, Cat\# P0705S),

18 and incubated for $2-\mathrm{hr}$ at $37^{\circ} \mathrm{C}$. Samples were cleaned using C18 tips (Pierce, Cat\# 87784), and

19 eluted in $75 \%$ mass spectrometry grade acetonitrile, $0.1 \%$ formic acid (TCI Chemicals). Sample

20 peptide concentration was then quantified using a colorimetric assay (Pierce, Cat\# 23275).

\section{Mass spectrometry data acquisition}

23 An aliquot of each sample equivalent to $3 \mu \mathrm{g}$ was loaded onto a trap column (Acclaim PepMap

24100 pre-column, $75 \mu \mathrm{m} \times 2 \mathrm{~cm}, \mathrm{C} 18,3 \mu \mathrm{m}, 100 \AA$, Thermo Scientific) connected to an analytical

25 column (Acclaim PepMap RSLC column C18 $2 \mu \mathrm{m}, 100 \AA ̊, 50 \mathrm{~cm} \times 75 \mu \mathrm{m}$ ID, Thermo Scientific)

26 using the autosampler of an Easy nLC 1000 (Thermo Scientific) with solvent A consisting of 0.1\%

27 formic acid in water and solvent B, $0.1 \%$ formic acid in acetonitrile. The peptide mixture was

28 gradient eluted into an Orbitrap Fusion mass spectrometer (Thermo Scientific) using a $180 \mathrm{~min}$

29 gradient from 5\%-40\%B (A: 0.1\% formic acid in water, B:0.1\% formic acid in acetonitrile) 
1 followed by a 20 min column wash with $100 \%$ solvent $B$. The full scan MS was acquired over range 400-1400 m/z with a resolution of 120,000 (@ m/z 200), AGC target of 5e5 charges and a maximum ion time of $100 \mathrm{~ms}$ and $2 \mathrm{~s}$ cycle time. Data dependent MS/MS scans were acquired in the linear ion trap using CID with a normalized collision energy 35\%. For quantitation of scans, synchronous precursor selection was used to select 10 most abundant product ions for subsequent $\mathrm{MS}^{\wedge} 3$ using AGC target 5e4 and fragmentation using HCD with NCE 55\% and resolution in the Orbitrap 60,000. Dynamic exclusion of each precursor ion for $30 \mathrm{~s}$ was employed. Data were analyzed using Proteome Discoverer 2.4.1 (Thermo Scientific).

Computational determination of the human $\mathrm{N}$-glycome and substrates analyses

11 The human $\mathrm{N}$-glycome was defined by the total predicted $\mathrm{N}$-glycosylated proteins from the

12 reviewed human proteome from the UniprotKB (accessed 8/10/2020). Both manual and

13 automated curation of the data set was preformed to remove mitochondrial proteins as well as

14 proteins smaller than 50 amino acids from the dataset. All annotations were derived directly

15 from the UniprotKB information and annotations available for these proteins were analyzed in

16 R. Determination of the pl values were performed by the $\mathrm{pl} / \mathrm{MW}$ tool on the Expasy database.

\section{Reglucosylation validation assay}

19 Five $10 \mathrm{~cm}$ plates were seeded with 3.5 million cells each and allowed to grow for $48 \mathrm{hr}$. Cells

20 were treated with DNJ at $500 \mu \mathrm{M}$ for $14 \mathrm{hr}$. Prior to lysis, the media was aspirated and cells

21 were washed once with filter sterilized PBS. Cells were lysed in $1 \mathrm{ml}$ of MNT (20 mM MES, 100

$22 \mathrm{mM} \mathrm{NaCl}, 30 \mathrm{mM}$ Tris $\mathrm{pH} 7.5,0.5 \%$ Triton X-100) with protease inhibitors (50 $\mu \mathrm{M}$ Calpain

23 inhibitor I, $1 \mu \mathrm{M}$ pepstatin, $10 \mu \mathrm{g} / \mathrm{ml}$ aprotinin, $10 \mu \mathrm{g} / \mathrm{ml}$ leupeptin, $400 \mu \mathrm{M}$ PMSF) and $20 \mathrm{mM}$

$24 \mathrm{~N}$-ethyl maleimide, shaken vigorously for $5 \mathrm{~min}$ at $4{ }^{\circ} \mathrm{C}$, and centrifuged for $5 \mathrm{~min}$ at $17,000 \mathrm{~g}$ at

$25 \quad 4{ }^{\circ} \mathrm{C} .50 \mu \mathrm{l}$ bed volume of glutathione beads was added to each pull-down and incubated for 1

$26 \mathrm{hr}$ at $4{ }^{\circ} \mathrm{C}$ under gentle rotation. Beads were then precipitated by centrifugation at $1,000 \mathrm{~g}$ for 5

$27 \min$ at $4{ }^{\circ} \mathrm{C}$. Supernatant was collected with $10 \%$ used for WCL and the remainder split evenly

28 between GST-CRT and GST-CRT-Y109A conjugated glutathione beads, which were generated as

29 previously described, and incubated for $16 \mathrm{hr}$ at $4{ }^{\circ} \mathrm{C}$ under gentle rotation. Beads were 
1 precipitated at $1,000 \mathrm{~g}$ for $5 \mathrm{~min}$ at $4{ }^{\circ} \mathrm{C}$. Supernatant was aspirated and beads were washed twice with lysis buffer without protease inhibitors. Beads were treated with reducing sample buffer (30 mM Tris-HCl pH 6.8, 9\% SDS, 15\% glycerol, 0.05\% bromophenol blue). WCLs were trichloroacetic acid (TCA) precipitated by adding TCA to cell lysate to a final concentration of $10 \%$. Cell lysate was then briefly rotated and allowed to incubate on ice for $15 \mathrm{~min}$ before centrifugation at $17,000 \mathrm{~g}$ for $10 \mathrm{~min}$ at $4{ }^{\circ} \mathrm{C}$. Supernatants were aspirated and washed twice with cold acetone and centrifuged at $17,000 \mathrm{~g}$ for $10 \mathrm{~min}$ at $4{ }^{\circ} \mathrm{C}$. Supernatants were aspirated and the remaining precipitant was allowed to dry for $5 \mathrm{~min}$ at room temperature and briefly at $65{ }^{\circ} \mathrm{C}$. Precipitated protein was resuspended in sample buffer. Samples were resolved on a $9 \%$ reducing SDS-PAGE and imaged by immunoblotting.

Metabolic labeling and IGF-1R immunoprecipitation

13 Two million cells were plated in $6 \mathrm{~cm}$ plates and allowed to grow for $40 \mathrm{hr}$. Cells were pulse 14 labeled for $1 \mathrm{hr}$ with $120 \mu \mathrm{Ci}$ of EasyTag Express ${ }^{35} \mathrm{~S}$ Protein Labeling Mix $\left[{ }^{35} \mathrm{~S}\right]-\mathrm{Cys} / \mathrm{Met}$

15 (PerkinElmer; Waltham, MA). Immediately after the radioactive pulse, cells were washed with PBS and either lysed in MNT with a protease inhibitor cocktail (Halt protease and phosphatase

17 inhibitor single-use cocktail, Thermo Fisher) and $20 \mathrm{mM} \mathrm{NEM}$, or chased for indicated time

18 using regular growth media. Where indicated, cells were treated with $500 \mu \mathrm{M}$ DNJ for 30 min 19 prior to $\left[{ }^{35} \mathrm{~S}\right]$-Cys/Met labeling and through the chase. Cell lysates were shaken for 5 min at 4 $20{ }^{\circ} \mathrm{C}$, centrifuged at $17,000 \mathrm{~g}$ for $5 \mathrm{~min}$ at $4{ }^{\circ} \mathrm{C}$, and the supernatants were collected. Samples 21 were pre-cleared with a $20 \mu \mathrm{l}$ bed volume of protein-A sepharose beads (GE Healthcare) by

22 end-over-end rotation for $1 \mathrm{hr}$ at $4{ }^{\circ} \mathrm{C}$. The supernatants were collected and incubated with a

$2330 \mu \mathrm{l}$ bed volume of protein-A-sepharose beads and $1.5 \mu \mathrm{l}$ of $\alpha$-IGF-1 receptor $\beta$ (D23H3) XP

24 (Cell Signaling) per sample. Samples were washed with MNT without protease inhibitors or 25 NEM and eluted in sample buffer. Samples were then resolved on a 9\% reducing SDS-PAGE, 26 imaged using a GE Typhoon FLA 9500 phosphorimager (GE Healthcare), and quantified using 27 ImageJ. 
1 Three million cells for each indicated cell line were plated in a $10-\mathrm{cm}$ plate and allowed to grow

2 for $48 \mathrm{hr}$. Cells were lysed in $300 \mu$ RIPA buffer (1\% SDS, 1\% NP-40, 0.5\% sodium deoxycholate,

$3150 \mathrm{mM} \mathrm{NaCl}, 50 \mathrm{mM}$ Tris- $\mathrm{HCl} \mathrm{pH}$ 8.0) with protease inhibitor cocktail and $20 \mathrm{mM} \mathrm{NEM}$.

4 Samples were then sonicated for $20-\mathrm{sec}$ at $40 \%$ amplitude (Sonics vibra cell VC130PB), shaken

5 vigorously for $5 \mathrm{~min}$, and centrifuged for $5 \mathrm{~min}$ at 17,000 g. $20 \mu \mathrm{l}$ of the resulting lysate was

6 heated at $95^{\circ} \mathrm{C}$ for $5 \mathrm{~min}$, and treated with either $10 \mu \mathrm{l}$ of PNGaseF or EndoH for $1 \mathrm{hr}$ at $37^{\circ} \mathrm{C}$,

7 according to the manufacturer's instructions (NEB). Samples were diluted 1:1 into sample

8 buffer and imaged by immunoblotting.

\section{RNAseq library preparation and Sequencing}

11 Three million cells for each indicated cell line were plated in $10 \mathrm{~cm}$ plates and allowed to grow

12 for $48 \mathrm{hr}$. Cells were then lysed in TRIzol buffer and RNA was isolated using RNA Clean

13 Concentrate Kit with in-column DNase-I treatment (Zymo Research Corp), following

14 manufacturer instructions. The quantity of RNA was assayed on Qubit using RNA BR assay (Life

15 Technologies Corp), and quality was assessed on Agilent 2100 Bioanalyzer using RNA 6000 Nano

16 Assay (Agilent Technologies Inc). Total RNA was used to isolate poly(A) mRNA using NEBNext

$17 \operatorname{Poly}(\mathrm{A})$ mRNA Magnetic Isolation Module, and libraries were prepared using NEBNext Ultrall

18 Directional RNA Library Prep Kit for Illumina (New England Biolabs) following manufacturer

19 instructions. The quantity of library was assayed using Qubit DNA HS assay (Life Technologies

20 Corp), and quality was analyzed on Bioanalyzer (Agilent Technologies Inc). Libraries were

21 sequenced on Illumina NextSeq 500 platform using NextSeq 500/550 High Output v2 kit (150

22 cycles) with 76 bp paired-end sequencing chemistry.

Sequence quality was assessed using FastQC (Andrews) and MultiQC (Ewels et al., 2016).

24 Reads were aligned to the hg38 human reference genome using STAR (Dobin et al., 2013).

25 Transcript abundance was quantified using RSEM (Li and Dewey, 2011) and normalized to

26 counts per million (CPM) in R using the edgeR software package (Robinson et al., 2010).

27 Analyses to compare gene expression between cell types was conducted in Excel by finding the

28 average CPM in the pool of genes of interest for the associated cell type and determining the

29 standard deviation away from the average for each gene of interest. 
ACKNOWLEDGEMENTS: This work was supported by the National Institutes of Health under award number (GM086874 (to D.N.H.); and a Chemistry-Biology Interface program training grant (T32GM008515 to B.M.A. and N.P.C.). Mass spectral data were obtained at the University of Massachusetts Mass Spectrometry Center (Director Dr. Steve Eyles). Flow cytometry and RNAseq data were conducted at the University of Massachusetts Flow Cytometry Core Facility

(Director Dr. Amy Burnside) and the Genomics Resource Laboratory (Director Dr. Ravi Ranjan), respectively. John Swenson conducted the analysis of raw RNAseq data.

\section{Declaration of Interests}

12 The authors declare no competing interests.

\section{REFERENCES}

Adams, B.M., Oster, M.E., and Hebert, D.N. (2019a). Protein Quality Control in the Endoplasmic Reticulum. Protein J 38.

Adams, B.M., Ke, H., Gierash, L.M., Gershenson, A., and Hebert, D.N. (2019b). Proper secretion of the serpin antithrombin relies strictly on thiol-dependent quality control. J Biol Chem 294, 18992-19011.

21 Aebi, M. (2013). N-linked protein glycosylation in the ER. Biochim Biophys Acta 1833, 2430222437.

Arnold, S.M., and Kaufman, R.J. (2003). The Noncatalytic Portion of Human UDP-glucose: Glycoprotein Glucosyltransferase I Confers UDP-glucose Binding and Transferase Function to the Catalytic Domain. J Biol Chem 278, 43320-43328. Enzymatic Activity. Biochemistry 39, 2149-2163. 
1 Balchin, D., Hayer-Hartl, M., and Hartl, U.F. (2016). In vivo aspects of protein folding and quality

2 control. Science 353, aac4354.

3 Balchin, D., Hayer-Hartl, M., and Hartl, F.U. (2020). Recent advances in understanding catalysis

4 of protein folding by molecular chaperones. FEBS Letters 594, 2770-2781.

5 Barlowe, C., and Helenius, A. (2016). Cargo Capture and Bulk Flow in the Early Secretory

6 Pathway. Annu Rev Cell Dev Biol 32, 197-222.

7 Beck, M., and Hurt, E. (2016). The nuclear pore complex: understanding its function through

8 structural insight. Nat Rev Mol Cell 18, 73-89.

9 Cacan, R., Duvet, S., Labiau, O., Verbert, A., and Krag, S.S. (2001). Monoglucosylated

10 oligomannosides are released during the degradation process of newly synthesized

11 glycoproteins. J Biol Chem 276, 22307-22312.

12 Caramelo, J.J., Castro, O.A., Prat-Gay, G. de, and Parodi, A.J. (2004). The endoplasmic reticulum 13 glucosyltransferase recognizes nearly native glycoprotein folding intermediates. J Biol Chem $14 \quad 279,46280-46285$.

15 Cesani, M., Lorioli, L., Grossi, S., Amico, G., Fumagalli, F., Spiga, I., Filocamo, M., and Biffi, A.

16 (2016). Mutation Update of ARSA and PSAP Genes Causing Metachromatic Leukodystrophy.

17 Hum Mutat 37, 16-27.

18 Chen, W., Helenius, J., Braakman, I., and Helenius, A. (1995). Cotranslational folding and

19 calnexin binding during glycoprotein synthesis. Proc Natl Acad Sci USA 92, 6229-6233.

20 Cherepanova, N.A., Shrimal, S., and Gilmore, R. (2016). N-linked glycosylation and homeostasis

21 of the endoplasmic reticulum. Current Opinion in Cell Biology 41, 57-65.

22 Cherepanova, N.A., Venev, S.V., Leszyk, J.D., Shaffer, S.A., and Gilmore, R. (2019). Quantitative

23 proteomics reveals new classes of STT3A- and STT3B-dependent N-glycosylation sites. J Cell Biol

$24218,2782-2796$.

25 Chng, W.J., Gualberto, A., and Fonseca, R. (2006). IGF-1R is overexpressed in poor-prognostic 26 subtypes of multiple myeloma. Leukemia 20, 174-176.

27 Daniels, R., Kurowski, B., Johnson, A.E., and Hebert, D.N. (2003). N-linked glycans direct the 28 cotranslational folding pathway of influenza hemagglutinin. Mol Cell 11, 79-90.

29 Dell'Angelica, E.C., and Payne, G.S. (2001). Intracellular Cycling of Lysosomal Enzyme Receptors: 30 Cytoplasmic Tails' Tales. Cell 106, 395-398.

31 Desbois-Mouthon, C., Wendum, D., Cadoret, A., Rey, C., Leneuve, P., Blaise, A., Housset, C., 32 Tronche, F., Le Bouc, Y., and Holzenberger, M. (2006). Hepatocyte proliferation during liver 
1 regeneration is impaired in mice with liver-specific IGF-1R knockout. The FASEB Journal 20,

$2773-775$.

3 Ferreira, C.R., and Gahl, W.A. (2017). Lysosomal storage diseases. Transl Sci Rare Dis 2, 1-71.

4 Ferris, S.P., Jaber, N.S., Molinari, M., Arvan, P., and Kaufman, R.J. (2013). UDP-

5 glucose:glycoprotein Glucosyltransferase (UGGT1) Promotes Substrate Solubility in the

6 Endoplasmic Reticulum. Mol Biol Cell 24, 2597-2608.

7 Hartl, U.F. (2017). Protein Misfolding Diseases. Annu Rev Biochem 86, 21-26.

8 Hebert, D.N., and Molinari, M. (2007). In and out of the ER: protein folding, quality control, 9 degradation, and related human diseases. Physiol Rev 87, 1377-1408.

10 Hebert, D.N., Foellmer, B., and Helenius, A. (1995). Glucose trimming and reglucosylation 11 determine glycoprotein association with calnexin in the endoplasmic reticulum. Cell 81, 42512433.

13 Hebert, D.N., Foellmer, B., and Helenius, A. (1996). Calnexin and calreticulin promote folding, 14 delay oligomerization and suppress degradation of influenza hemagglutinin in microsomes.

15 EMBO J 15, 2961-2968.

16 Hebert, D.N., Lamriben, L., Powers, E.T., and Kelly, J.W. (2014). The intrinsic and extrinsic effects 17 of N-linked glycans on glycoproteostasis. Nat Chem Biol 10, 902-910.

18 Helenius, A. (1994). How N-linked oligosaccharides affect glycoprotein folding in the 19 endoplasmic reticulum. Mol Biol Cell 5, 253-265.

20 Helenius, A., and Aebi, M. (2004). Roles of N-linked glycans in the endoplasmic reticulum. Annu 21 Rev Biochem 73, 1019-1049.

22 Helenius, A., and Hammond, C. (1994).

23 Folding of VSV G protein: sequential interaction with BiP and calnexin. Science 266, 456-458.

24 Houry, W.A., Frishman, D., Eckerskorn, C., Lottspeich, F., and Hartl, F.U. (1999). Identification of 25 in vivo substrates of the chaperonin GroEL. Nature 402, 147-154.

26 Itzhak, D.N., Tyanova, S., Cox, J., and Borner, G.H. (2016). Global, Quantitative and Dynamic 27 Mapping of Protein Subcellular Localization. ELife 5.

28 Kapoor, M., Ellgaard, L., Gopalakrishnapai, J., Schirra, C., Gemma, E., Oscarson, S., Helenius, A., 29 and Surolia, A. (2004). Mutational analysis provides molecular insight into the carbohydrate30 binding region of calreticulin: pivotal roles of tyrosine-109 and aspartate-135 in carbohydrate 31 recognition. Biochemistry 43, 97-106. 
1 Katta, S.S., Smoyer, C.J., and Jaspersen, S.L. (2014). Destination: inner nuclear membrane.

2 Trends Cell Biol 24, 221-229.

3 Kerner, M.J., Naylor, D.J., Ishihama, Y., Maier, T., Chang, H.-C., Stines, A.P., Georgopoulos, C., 4 Frishman, D., Hayer-Hartl, M., Mann, M., et al. (2005). Proteome-wide Analysis of Chaperonin5 Dependent Protein Folding in Escherichia coli. Cell 122, 209-220.

King, H., Aleksic, T., Haluska, P., and Macaulay, V.M. (2014). Can we unlock the potential of IGF$1 R$ inhibition in cancer therapy? Cancer Treatment Reviews 40, 1096-1105.

Kozlov, G., and Gehring, K. (2020). Calnexin Cycle - Structural Features of the ER Chaperone System. FEBS J doi: 10.1111/febs.15330. Online ahead of print.

10 Lamriben, L., Graham, J.B., Adams, B.M., and Hebert, D.N. (2016). N-glycan based ER molecular 11 chaperone and protein quality control system: the calnexin binding cycle. Traffic 17, 308-326.

Lehmann, M., André, F., Bellan, C., Remacle-Bonnet, M., Garrouste, F., Parat, F., Lissitsky, J.-C., Marvaldi, J., and Pommier, G. (1998). Deficient Processing and Activity of Type I Insulin-Like Growth Factor Receptor in the Furin-Deficient LoVo-C5 Cells*. Endocrinology 139, 3763-3771.

Lonowski, L.A., Narimatsu, Y., Riaz, A., Delay, C.E., Yang, Z., Niola, F., Duda, K., Ober, E.A., Clausen, H., Wandall, H.H., et al. (2017). Genome editing using FACS enrichment of nucleaseexpressing cells and indel detection by amplicon analysis. Nat Protoc 12, 581-603.

Mahuran, D.J. (1999). Biochemical consequences of mutations causing the GM2 gangliosidoses. Biochim Biophys Acta 1455, 105-138.

Mahuran, D.J., Neote, K., Klavins, M.H., Leung, A., and Gravel, R.A. (1988). Proteolytic processing of pro-alpha and pro-beta precursors from human beta-hexosaminidase. Generation of the mature alpha and beta a beta b subunits. J Biol Chem 263, 4612-4618.

Margittai, É., and Sitia, R. (2011). Oxidative Protein Folding in the Secretory Pathway and Redox 24 Signaling Across Compartments and Cells. Traffic 12, 1-8.

Molinari, M., Calanca, V., Galli, C., Lucca, P., and Paganetti, P. (2003). Role of EDEM in the release of misfolded glycoproteins from the calnexin cycle. Science 299, 1397-1400.

Molinari, M., Galli, C., Vanoni, O., Arnold, S.M., and Kaufman, R.J. (2005). Persistent glycoprotein misfolding activates the glucosidase II/UGT1-driven calnexin cycle to delay aggregation and loss of folding competence. Mol Cell 20, 503-512.

30 Mutgan, A.C., Besikcioglu, H.E., Wang, S., Friess, H., Ceyhan, G.O., and Demir, I.E. (2018).

31 Insulin/IGF-driven cancer cell-stroma crosstalk as a novel therapeutic target in pancreatic 32 cancer. Mol Cancer 17, 66-66. 
1 Narimatsu, Y., Joshi, H.J., Yang, Z., Gomes, C., Chen, Y.-H., Lorenzetti, F.C., Furukawa, S.,

2 Schjoldager, K.T., Hansen, L., Clausen, H., et al. (2018). A validated gRNA library for CRISPR/Cas9

3 targeting of the human glycosyltransferase genome. Glycobiology 28, 295-305.

4 Oda, Y., Hosokawa, N., Wada, I., and Nagata, K. (2003). EDEM as an acceptor of terminally

5 misfolded glycoproteins released from calnexin. Science 299, 1394-1397.

6 Parodi, A.J., and Caramelo, J.J. (2015). A sweet code for glycoprotein folding. FEBS Lett 589,

$7 \quad 3379-3387$.

8 Parodi, A.J., and Cazzulo, J.J. (1982). Protein glycosylation in Trypanosoma cruzi. II. Partial

9 characterization of protein-bound oligosaccharides labeled "in vivo." J Biol Chem 257, 7641-

107645.

11 Pearse, B.R., Gabriel, L., Wang, N., and Hebert, D.N. (2008). A cell-based reglucosylation assay

12 demonstrates the role of GT1 in the quality control of a maturing glycoprotein. J Cell Biol 181,

13 309-320.

14 Pearse, B.R., Tamura, T., Sunryd, J.C., Grabowski, G.A., Kaufman, R.J., and Hebert, D.N. (2010).

15 The role of UDP-Glc:glycoprotein glucosyltransferase 1 in the maturation of an obligate

16 substrate prosaposin. J Cell Biol 189, 829-841.

17 Quellhorst, G.J., Jr., O’Rear, J.L., Cacan, R., Verbert, A., and Krag, S.S. (1999). Nonglucosylated

18 oligosaccharides are transferred to protein in MI8-5 Chinese hamster ovary cells. Glycobiology

19 9, 65-72.

20 Rajagopalan, S., Xu, Y., and Brenner, M.B. (1994). Retention of Unassembled Components of

21 Integral Membrane Proteins by Calnexin. Science 263, 387-390.

22 Rauniyar, N., and Yates, J.R.I. (2014). Isobaric labeling-based relative quantification in shotgun

23 proteomics. J Proteome Res 13, 5293-5309.

24 Rebeaud, M.E., Mallik, S., Goloubinoff, P., and Tawfik, D.S. (2020). On the evolution of

25 chaperones and co-chaperones and the expansion of proteomes across the Tree of Life. BioRxiv

$26 \quad 2020.06 .08 .140319$.

27 Ritter, C., and Helenius, A. (2000). Recognition of local glycoprotein misfolding by the ER folding

28 sensor UDP-glucose:glycoprotein glucosyltransferase. Nat Struct Biol 7, 278-280.

29 Roversi, P., Marti, L., Caputo, A.T., Alonzi, D.S., Hill, J.C., Dent, K.C., Kumar, A., Levasseur, M.D., 30 Lia, A., Waksman, T., et al. (2017). Interdomain conformational flexibility underpins the activity 31 of UGGT, the eukaryotic glycoprotein secretion checkpoint. Proc Natl Acad Sci USA 114, 8544328549.

33 Ruiz-Canada, C., Kelleher, D.J., and Gilmore, R. (2009). Cotranslational and Posttranslational N34 glycosylation of Polypeptides by Distinct Mammalian OST Isoforms. Cell 136, 272-283. 
Satoh, T., Song, C., Zhu, T., Toshimori, T., Murata, K., Hayashi, Y., Kamikubo, H., Uchihashi, T., and Kato, K. (2017). Visualization of a flexible modular structure of the ER folding-sensor enzyme UGGT. Sci Rep 7, 12142.

Sell, C., Dumenil, G., Deveaud, C., Miura, M., Coppola, D., DeAngelis, T., Rubin, R., Efstratiadis, A., and Baserga, R. (1994). Effect of a null mutation of the insulin-like growth factor I receptor gene on growth and transformation of mouse embryo fibroblasts. Mol. Cell. Biol. 14, 3604.

Shrimal, S., and Gilmore, R. (2015). Reduced expression of the oligosaccharyltransferase exacerbates protein hypoglycosylation in cells lacking the fully assembled oligosaccharide donor. Glycobiology 25, 774-783.

10 Shrimal, S., Cherepanova, N.A., Mandon, E.S., Venev, S.V., and Gilmore, R. (2019). Asparagine11 linked Glycosylation Is Not Directly Coupled to Protein Translocation Across the Endoplasmic 12 Reticulum in Saccharomyces cerevisiae. Mol Biol Cell 30, 2626-2638.

13 Soldà, T., Galli, C., Kaufman, R.J., and Molinari, M. (2007). Substrate-specific requirements for 14 UGT1-dependent release from calnexin. Mol Cell 27, 238-249.

Sousa, M., and Parodi, A.J. (1995). The molecular basis for the recognition of misfolded glycoproteins by the UDP-Glc:glycoprotein glucosyltransferase. EMBO J 14, 4196-4203.

17 Takeda, Y., Seko, A., Hachisu, M., Daikoku, S., Izumi, M., Koizumi, A., Fujikawa, K., Kajihara, Y., 18 and Ito, Y. (2014). Both isoforms of human UDP-glucose:glycoprotein glucosyltransferase are 19 enzymatically active. Glycobiology $24,344-350$.

20 Tannous, A., Patel, N., Tamura, T., and Hebert, D.N. (2015). Reglucosylation by UDP-

21 glucose:glycoprotein glucosyltransferase 1 delays glycoprotein secretion but not degradation.

22 Mol Biol Cell 26, 390-405.

23 Taylor, S.C., Thibault, P., Tessier, D.C., Bergeron, J.J., and Thomas, D.Y. (2003). Glycopeptide 24 specificity of the secretory protein folding sensor UDP-glucose glycoprotein:glucosyltransferase. 25 EMBO Rep 4, 405-411.

26 Tom, R., Bisson, L., and Durocher, Y. (2008). Culture of HEK293-EBNA1 Cells for Production of 27 Recombinant Proteins. CSH Protoc pdb.prot4976.

28 Uhlén, M., Fagerberg, L., Hallström, B.M., Lindskog, C., Oksvold, P., Mardinoglu, A., Sivertsson, 29 Å., Kampf, C., Sjöstedt, E., Asplund, A., et al. (2015). Tissue-based map of the human proteome. 30 Science $347,1260419$.

31 Wang, N., Glidden, E.J., Murphy, S.R., Pearse, B.R., and Hebert, D.N. (2008). The cotranslational 32 maturation program for the type II membrane glycoprotein influenza neuraminidase. J Biol 33 Chem 283, 33826-33837. 
bioRxiv preprint doi: https://doi.org/10.1101/2020.10.15.340927; this version posted October 15,2020 . The copyright holder for this preprint (which was not certified by peer review) is the author/funder, who has granted bioRxiv a license to display the preprint in perpetuity. It is made available under aCC-BY-NC-ND 4.0 International license.

1 Zielinska, D.F., Gnad, F., Wiśniewski, J.R., and Mann, M. (2010). Precision Mapping of an In Vivo

2 N-Glycoproteome Reveals Rigid Topological and Sequence Constraints. Cell 141, 897-907.

3

4

5

6

7

8

9

10

11

12

13

14

15

16

17

18

19

20

21 